\title{
GAUSS-MANIN CONNECTIONS FOR ARRANGEMENTS, II NONRESONANT WEIGHTS
}

\author{
DANIEL C. COHEN ${ }^{\dagger}$ AND PETER ORLIK ${ }^{\ddagger}$
}

\begin{abstract}
We study the Gauss-Manin connection for the moduli space of an arrangement of complex hyperplanes in the cohomology of a nonresonant complex rank one local system. Aomoto and Kita determined this GaussManin connection for arrangements in general position. We use their results and an algorithm constructed in this paper to determine this Gauss-Manin connection for all arrangements.
\end{abstract}

\section{INTRODUCTION}

Let $\mathcal{A}=\left\{H_{1}, \ldots, H_{n}\right\}$ be an arrangement of $n$ ordered hyperplanes in $\mathbb{C}^{\ell}$, and let $\mathcal{L}$ be a local system of coefficients on $\mathrm{M}=M(\mathcal{A})=\mathbb{C}^{\ell} \backslash \bigcup_{j=1}^{n} H_{j}$, the complement of $\mathcal{A}$. The need to calculate the local system cohomology $H^{*}(\mathrm{M} ; \mathcal{L})$ arises in various contexts. For instance, local systems may be used to study the Milnor fiber of the non-isolated hypersurface singularity at the origin obtained by coning the arrangement, see [7, 5]. In mathematical physics, local systems on complements of arrangements arise in the Aomoto-Gelfand theory of multivariable hypergeometric integrals [2, 12, 18] and the representation theory of Lie algebras and quantum groups. These considerations lead to solutions of the Knizhnik-Zamolodchikov differential equation from conformal field theory, see [21, 23]. Here a central problem is the determination of the Gauss-Manin connection on $H^{*}(\mathrm{M} ; \mathcal{L})$ for certain arrangements, and certain local systems.

A complex rank one local system on $\mathrm{M}$ is determined by a collection of weights $\lambda=\left(\lambda_{1}, \ldots, \lambda_{n}\right) \in \mathbb{C}^{n}$. Associated to $\lambda$, we have a representation $\rho: \pi_{1}(\mathrm{M}) \rightarrow \mathbb{C}^{*}$, given by $\gamma_{j} \mapsto \exp \left(-2 \pi \mathrm{i} \lambda_{j}\right)$ for any meridian loop $\gamma_{j}$ about the hyperplane $H_{j}$ of $\mathcal{A}$, and an associated rank one local system $\mathcal{L}$ on $\mathrm{M}$. Several authors have considered Gauss-Manin connections with various conditions on the weights: Aomoto [1], Schechtman and Varchenko [21, 23, and Kaneko [15 studied discriminantal arrangements; Kanarek 14 studied the connection arising when a single hyperplane in the arrangement is allowed to move; Aomoto and Kita [2] determined the GaussManin connection matrices for general position arrangements; and Terao 22 computed these connection matrices for a larger class of arrangements. In this paper, we determine Gauss-Manin connection matrices for all arrangements and all weights $\lambda$ which satisfy the nonresonance condition (STV) of [20] stated in Theorem 6.1.

Date: July 4, 2002.

2000 Mathematics Subject Classification. 32S22, 14D05, 52C35, 55N25.

Key words and phrases. hyperplane arrangement, local system, Gauss-Manin connection.

${ }^{\dagger}$ Partially supported by Louisiana Board of Regents grant LEQSF(1999-2002)-RD-A-01 and by National Security Agency grant MDA904-00-1-0038.

${ }^{\ddagger}$ Partially supported by National Security Agency grant MDA904-02-1-0019. 
Given weights $\lambda$, we used stratified Morse theory in [3, 4] to construct a complex $K^{\bullet}(\mathcal{A})$ which computes $H^{*}(\mathrm{M} ; \mathcal{L})$. This construction is reviewed in Section 2 . In Section 3 , we compactify $\mathcal{A}$ by adding the infinite hyperplane $H_{n+1}$ to obtain the projective closure $\mathcal{A}_{\infty} \subseteq \mathbb{C P}^{\ell}$. Two arrangements are said to have the same combinatorial type if there is an order-preserving isomorphism between their intersection posets. If $\mathcal{T}$ is the combinatorial type of $\mathcal{A}$, we denote by $\operatorname{ind}(\mathcal{T})$ and $\operatorname{dep}(\mathcal{T})$ the independence and dependence sets of $\mathcal{T}$. These consist of all $\ell+1$ element subsets of $[n+1]=\{1,2, \ldots, n+1\}$ for which the corresponding hyperplanes in $\mathcal{A}_{\infty}$ are independent, and dependent, respectively.

The sets $\operatorname{ind}(\mathcal{T})$ and $\operatorname{dep}(\mathcal{T})$ may be used to describe the moduli space of all arrangements of type $\mathcal{T}$, defined and investigated by Terao in 222 . Let $\mathrm{B}(\mathcal{T})$ be a smooth, connected component of this moduli space. There is a fiber bundle $\pi: \mathrm{M}(\mathcal{T}) \rightarrow \mathrm{B}(\mathcal{T})$. The fibers of this bundle, $\pi^{-1}(\mathbf{x})=\mathrm{M}_{\mathbf{x}}$, are complements of arrangements $\mathcal{A}_{\mathbf{x}}$ of type $\mathcal{T}$, so they are diffeomorphic to $\mathrm{M}$ since $\mathrm{B}(\mathcal{T})$ is connected. Since the bundle $\pi: M(\mathcal{T}) \rightarrow B(\mathcal{T})$ is locally trivial, there is an associated vector bundle $\pi^{q}: \mathbf{H}^{q} \rightarrow \mathrm{B}(\mathcal{T})$, with fiber $\left(\pi^{q}\right)^{-1}(\mathbf{x})=H^{q}\left(\mathrm{M}_{\mathbf{x}} ; \mathcal{L}_{\mathbf{x}}\right)$ at $\mathbf{x} \in \mathrm{B}(\mathcal{T})$ for each $q, 0 \leq q \leq \ell$. The transition functions of this vector bundle are locally constant. Fixing a basepoint $\mathrm{x} \in \mathrm{B}(\mathcal{T})$, the operation of parallel translation of fibers over curves in $\mathrm{B}(\mathcal{T})$ in the vector bundle $\pi^{q}: \mathbf{H}^{q} \rightarrow \mathrm{B}(\mathcal{T})$ provides a complex representation

$$
\Psi_{\mathcal{T}}^{q}: \pi_{1}(\mathrm{~B}(\mathcal{T}), \mathbf{x}) \longrightarrow \operatorname{Aut}_{\mathbb{C}}\left(H^{q}\left(\mathrm{M}_{\mathbf{x}} ; \mathcal{L}_{\mathbf{x}}\right)\right) .
$$

A key idea in this paper is to extract information about arbitrary arrangements using information about general position arrangements. Let $\mathcal{G}$ denote the combinatorial type of a general position arrangement of $n$ hyperplanes in $\mathbb{C}^{\ell}$, so $\operatorname{dep}(\mathcal{G})=\emptyset$. Let $\mathrm{B}(\mathcal{G})$ be its moduli space, $\mathbf{y} \in \mathrm{B}(\mathcal{G}), \mathcal{B}_{\mathrm{y}}^{n, \ell}$ the corresponding arrangement, with complement $\mathrm{E}_{\mathbf{y}}^{n, \ell}=M\left(\mathcal{B}_{\mathbf{y}}^{n, \ell}\right)$. In Section $\mathbb{G}$, we compare the representation (1.1) with the analogous representation $\Psi_{\mathcal{G}}^{q}: \pi_{1}(\mathrm{~B}(\mathcal{G}), \mathbf{y}) \rightarrow \operatorname{Aut}_{\mathbb{C}}\left(H^{q}\left(\mathrm{E}_{\mathbf{y}}^{n, \ell} ; \mathcal{L}_{\mathbf{y}}\right)\right)$. To this end, we introduce a space $\mathrm{Y}(\mathcal{T})$ which contains $\mathrm{B}(\mathcal{T})$ and $\mathrm{B}(\mathcal{G})$ as disjoint subspaces. Let $i_{\mathcal{T}}: \mathrm{B}(\mathcal{G}) \rightarrow \mathrm{Y}(\mathcal{T})$ and $j_{\mathcal{T}}: \mathrm{B}(\mathcal{T}) \rightarrow \mathrm{Y}(\mathcal{T})$ denote the natural inclusions. Given $\gamma \in \pi_{1}(\mathrm{~B}(\mathcal{T}), \mathbf{x})$, we show that there is a compatible $\Gamma \in \pi_{1}(\mathrm{~B}(\mathcal{G}), \mathbf{y})$, a loop for which the homology classes $[\gamma]$ and $[\Gamma]$ satisfy $\left(i_{\mathcal{T}}\right)_{*}([\Gamma])=\left(j_{\mathcal{T}}\right)_{*}([\gamma])$. For compatible loops, the automorphisms $\Psi_{\mathcal{T}}^{q}(\gamma)$ and $\Psi_{\mathcal{G}}^{q}(\Gamma)$ are related by

$$
\bar{s}_{\mathbf{x}}^{q} \circ \Psi_{\mathcal{G}}^{q}(\Gamma) \sim \Psi_{\mathcal{T}}^{q}(\gamma) \circ \bar{s}_{\mathbf{x}}^{q} .
$$

Here $\bar{s}_{\mathbf{x}}^{q}: H^{q}\left(M\left(\mathcal{B}_{\mathbf{y}}^{n, \ell}\right) ; \mathcal{L}_{\mathbf{y}}\right) \rightarrow H^{q}\left(M\left(\mathcal{A}_{\mathbf{x}}\right) ; \mathcal{L}_{\mathbf{x}}\right)$ is the map in local system cohomology induced by a certain chain map $s_{\mathbf{x}}^{\bullet}: K^{\bullet}\left(\mathcal{B}_{\mathbf{y}}^{n, \ell}\right) \rightarrow K^{\bullet}\left(\mathcal{A}_{\mathbf{x}}\right)$, and $\sim$ denotes equality up to conjugacy.

These compatible classes are studied further in Section 5 . The moduli space $\mathrm{B}(\mathcal{G})$ of general position arrangements is the complement of a divisor $\mathrm{D}(\mathcal{G})=\bigcup_{J} \mathrm{D}_{J}$ in $\left(\mathbb{C P}^{\ell}\right)^{n}$. The components $\mathrm{D}_{J}$ of this divisor are irreducible hypersurfaces indexed by $\ell+1$ element subsets $J$ of $[n+1]$. For each such $J$, let $\Gamma_{J}$ be a meridian loop in $\mathrm{B}(\mathcal{G})$ about a generic point in $\mathrm{D}_{J}$. We prove that the homology group $H_{1}(\mathrm{~B}(\mathcal{G})$ ) is generated by the classes $\left[\Gamma_{J}\right]$. Note that $\Gamma_{J}$ is a loop in $Y(\mathcal{T})$ for any combinatorial type $\mathcal{T}$ since $\mathrm{B}(\mathcal{G}) \subseteq \mathrm{Y}(\mathcal{T})$. We say that the type $\mathcal{T}$ covers $\mathcal{T}^{\prime}$ if $\mathrm{B}\left(\mathcal{T}^{\prime}\right)$ has complex codimension one in the closure $\overline{\mathrm{B}}(\mathcal{T})$. If $\gamma \in \pi_{1}(\mathrm{~B}(\mathcal{T}), \mathbf{x})$ is a simple loop in $\mathrm{B}(\mathcal{T})$ 
about a generic point in $\mathrm{B}\left(\mathcal{T}^{\prime}\right)$, we show that the homology class $[\gamma]$ satisfies

$$
\left(j_{\mathcal{T}}\right)_{*}([\gamma])=\sum_{J \in \operatorname{dep}\left(\mathcal{T}^{\prime}, \mathcal{T}\right)} m_{J} \cdot\left[\Gamma_{J}\right]
$$

where $\operatorname{dep}\left(\mathcal{T}^{\prime}, \mathcal{T}\right)=\operatorname{dep}\left(\mathcal{T}^{\prime}\right) \backslash \operatorname{dep}(\mathcal{T})$, and $m_{J}$ is the order of vanishing of the restriction of a defining polynomial for $\mathrm{D}_{J}$ to $\overline{\mathrm{B}}(\mathcal{T})$ along $\mathrm{B}\left(\mathcal{T}^{\prime}\right)$.

If $\mathcal{L}$ is a nontrivial rank one local system on the complement $\mathrm{E}^{n, \ell}$ of a general position arrangement of $n$ hyperplanes in $\mathbb{C}^{\ell}$, then the cohomology $H^{q}\left(\mathrm{E}^{n, \ell} ; \mathcal{L}\right)$ vanishes in all dimensions $q$ except possibly $q=\ell$. For an arbitrary arrangement $\mathcal{A}$, a collection of weights $\lambda$ and the corresponding local system $\mathcal{L}$ are called nonresonant if the cohomology $H^{q}(\mathrm{M} ; \mathcal{L})$ vanishes in all dimensions $q$ except possibly $q=\ell$. We review the (STV)-condition for $\mathcal{T}$-nonresonance [20] and the $\beta \mathbf{n b c}$ basis for the nonvanishing group 11] in Section 6. We show that for $\mathcal{T}$-nonresonant weights $\lambda$, the chain $\operatorname{map} s^{\bullet}: K^{\bullet}\left(\mathcal{B}^{n, \ell}\right) \rightarrow K^{\bullet}(\mathcal{A})$ induces a surjection $\bar{s}^{\ell}: H^{\ell}\left(\mathrm{E}^{n, \ell}, \mathcal{L}\right) \rightarrow H^{\ell}(\mathrm{M} ; \mathcal{L})$.

The vector bundle $\pi^{q}: \mathbf{H}^{q} \rightarrow \mathrm{B}(\mathcal{T})$, with fiber $\left(\pi^{q}\right)^{-1}(\mathbf{x})=H^{q}\left(\mathrm{M}_{\mathbf{x}} ; \mathcal{L}_{\mathbf{x}}\right)$ at $\mathbf{x} \in \mathrm{B}(\mathcal{T})$, supports a Gauss-Manin connection corresponding to the representation (1.1). We study this connection in Section 7 for $\mathcal{T}$-nonresonant weights, where only $q=\ell$ is relevant. Let $\gamma \in \pi_{1}(\mathrm{~B}(\mathcal{T}), \mathbf{x})$ be represented by $g: \mathbb{S}^{1} \rightarrow \mathrm{B}(\mathcal{T})$. A connection matrix, $\Omega_{\mathcal{T}}(\gamma)$, for the induced connection on the pullback of the vector bundle $\pi^{\ell}: \mathbf{H}^{\ell} \rightarrow \mathrm{B}(\mathcal{T})$ along $g$ is called a Gauss-Manin connection matrix. Let $\Gamma \in \pi_{1}(\mathrm{~B}(\mathcal{G}), \mathbf{y})$ be a loop whose homology class is given by $(1.3)$ above, $[\Gamma]=$ $\sum_{J \in \operatorname{dep}\left(\mathcal{T}^{\prime}, \mathcal{T}\right)} m_{J} \cdot\left[\Gamma_{J}\right]$. Then $\gamma$ and $\Gamma$ are compatible, and it follows from (1.2) that $\bar{s}_{\mathbf{x}}^{\ell} \circ \Psi_{\mathcal{G}}^{\ell}(\Gamma) \sim \Psi_{\mathcal{T}}^{\ell}(\gamma) \circ \bar{s}_{\mathbf{x}}^{\ell}$. Since the homomorphism $\bar{s}_{\mathbf{x}}^{\ell}: H^{\ell}\left(\mathrm{E}_{\mathbf{y}}^{n, \ell}, \mathcal{L}_{\mathbf{y}}\right) \rightarrow$ $H^{\ell}\left(\mathrm{M}_{\mathbf{x}} ; \mathcal{L}_{\mathbf{x}}\right)$ is surjective for $\mathcal{T}$-nonresonant weights, this determines the GaussManin connection matrix $\Omega_{\mathcal{T}}(\gamma)$.

Aomoto and Kita 2] obtained explicit formulas for the Gauss-Manin connection matrices $\Omega_{\mathcal{G}}\left(\Gamma_{J}\right)$ in the case of general position arrangements, see also 18 . We combine our results with these formulas to obtain the main result of this paper. It is a combinatorial algorithm for calculating Gauss-Manin connection matrices for an arbitrary combinatorial type $\mathcal{T}$, and $\mathcal{T}$-nonresonant weights, in terms of those for the type $\mathcal{G}$ of general position arrangements. Let $\mathcal{T}$ be a combinatorial type which covers the type $\mathcal{T}^{\prime}$. Let $\gamma \in \pi_{1}(\mathrm{~B}(\mathcal{T}), \mathbf{x})$ a simple loop in $\mathrm{B}(\mathcal{T})$ about a generic point in $\mathrm{B}\left(\mathcal{T}^{\prime}\right)$. We agree to write $\Omega_{\mathcal{T}}\left(\mathcal{T}^{\prime}\right)$ in place of $\Omega_{\mathcal{T}}(\gamma)$ in this situation. Similarly, we write $\Omega_{\mathcal{G}}(J)$ in place of $\Omega_{\mathcal{G}}\left(\Gamma_{J}\right)$. Fix $\mathcal{T}$-nonresonant (and hence $\mathcal{G}$ nonresonant) weights $\lambda$. For $\mathbf{x} \in \mathrm{B}(\mathcal{T})$ and $\mathbf{y} \in \mathrm{B}(\mathcal{G})$, the corresponding local system cohomology groups of the fibers, $H^{q}\left(\mathrm{M}_{\mathbf{x}} ; \mathcal{L}_{\mathbf{x}}\right)$ and $H^{q}\left(\mathrm{E}_{\mathbf{y}}^{n, \ell} ; \mathcal{L}_{\mathbf{y}}\right)$, depend only on the combinatorial types $\mathcal{T}$ and $\mathcal{G}$ and vanish for $q \neq \ell$. So we write $H^{\ell}\left(\mathrm{M}_{\mathbf{x}} ; \mathcal{L}_{\mathbf{x}}\right)=$ $H^{\ell}(\mathcal{T})$ and $H^{\ell}\left(\mathrm{E}_{\mathbf{y}}^{n, \ell} ; \mathcal{L}_{\mathbf{y}}\right)=H^{\ell}(\mathcal{G})$. This notation makes it clear that the GaussManin connection matrix $\Omega_{\mathcal{T}}\left(\mathcal{T}^{\prime}\right)$ depends only on the combinatorial types involved. The matrix $\Omega_{\mathcal{T}}\left(\mathcal{T}^{\prime}\right)$ is obtained by the combinatorial formula recorded below.

Theorem. Let $\mathcal{T}$ be a combinatorial type which covers the type $\mathcal{T}^{\prime}$. Let $\lambda$ be a collection of $\mathcal{T}$-nonresonant weights, and let $P(\mathcal{T})$ be the matrix of the surjection $H^{\ell}(\mathcal{G}) \rightarrow H^{\ell}(\mathcal{T})$ in the respective $\beta$ nbc bases. Then a Gauss-Manin connection matrix $\Omega_{\mathcal{T}}\left(\mathcal{T}^{\prime}\right)$ is determined by the matrix equation

$$
P(\mathcal{T}) \cdot \Omega_{\mathcal{T}}\left(\mathcal{T}^{\prime}\right)=\left(\sum_{J \in \operatorname{dep}\left(\mathcal{T}^{\prime}, \mathcal{T}\right)} m_{J} \cdot \Omega_{\mathcal{G}}(J)\right) \cdot P(\mathcal{T})
$$

Illustrations of the algorithm provided by this result are given in Section 8 . 


\section{Preliminary Results}

In this section, we record a number of results concerning a fixed arrangement. We use notation and results from [17, 18].

Let $\mathcal{A}=\left\{H_{1}, \ldots, H_{n}\right\}$ be an arrangement of $n$ ordered hyperplanes in $\mathbb{C}^{\ell}$. Assume that the first $\ell$ hyperplanes of $\mathcal{A}$ are linearly independent, so $\mathcal{A}$ is essential. Choose coordinates $u_{1}, \ldots, u_{\ell}$ for $\mathbb{C}^{\ell}$, and for each hyperplane $H_{j}$ of $\mathcal{A}$, let $\alpha_{j}=\alpha_{j}\left(u_{1}, \ldots, u_{\ell}\right)$ be a linear polynomial such that $H_{j}$ is defined by the vanishing of $\alpha_{j}$. Then $Q(\mathcal{A})=\prod_{j=1}^{n} \alpha_{j}\left(u_{1}, \ldots, u_{\ell}\right)$ is a defining polynomial for $\mathcal{A}$, and the complement $\mathrm{M}=M(\mathcal{A})$ is given by $\mathrm{M}=\mathbb{C}^{\ell} \backslash \bigcup_{j=1}^{n} H_{j}=\mathbb{C}^{\ell} \backslash Q(\mathcal{A})^{-1}(0)$.

Choose coordinates $u_{1}, \ldots, u_{n}$ for $\mathbb{C}^{n}$, and consider the arrangement $\mathcal{B}^{n}$ in $\mathbb{C}^{n}$ defined by $Q\left(\mathcal{B}^{n}\right)=\prod_{j=1}^{\ell} \alpha_{j}\left(u_{1}, \ldots, u_{\ell}\right) \prod_{j=\ell+1}^{n} u_{j}$. Since the first $\ell$ hyperplanes of $\mathcal{A}$ are linearly independent, $\mathcal{B}^{n}$ is an arrangement of $n$ linearly independent hyperplanes in $\mathbb{C}^{n}$, a Boolean arrangement, and the complement $\mathrm{E}^{n}=M\left(\mathcal{B}^{n}\right)=$ $\left(\mathbb{C}^{*}\right)^{n}$ is the complex $n$-torus. Define $s: \mathbb{C}^{\ell} \rightarrow \mathbb{C}^{n}$ by

$$
s\left(u_{1}, \ldots, u_{\ell}\right)=\left(u_{1}, \ldots, u_{\ell}, \alpha_{\ell+1}\left(u_{1}, \ldots, u_{\ell}\right), \ldots, \alpha_{n}\left(u_{1}, \ldots, u_{\ell}\right)\right) .
$$

Then $s: \mathrm{M} \rightarrow \mathrm{E}^{n}$, and we have the following.

Proposition 2.1. Let $\mathcal{A}$ be an essential arrangement of $n$ hyperplanes in $\mathbb{C}^{\ell}$. Then the complement $\mathrm{M}$ of $\mathcal{A}$ is an affine section of the complex $n$-torus $\mathrm{E}^{n}$.

Let $A(\mathcal{A})$ be the Orlik-Solomon algebra of $\mathcal{A}$, generated by one dimensional classes $a_{j}, 1 \leq j \leq n$. It is the quotient of the exterior algebra generated by these classes by a homogeneous ideal, hence a finite dimensional graded $\mathbb{C}$-algebra. There is an isomorphism of graded algebras $H^{*}(\mathrm{M} ; \mathbb{C}) \simeq A(\mathcal{A})$. In particular, $\operatorname{dim} A^{q}(\mathcal{A})=b_{q}(\mathcal{A})$ where $b_{q}(\mathcal{A})=\operatorname{dim} H^{q}(\mathrm{M} ; \mathbb{C})$ denotes the $q$-th Betti number of $\mathrm{M}$ with trivial local coefficients $\mathbb{C}$.

This construction is realized topologically by the map $s: \mathrm{M} \rightarrow \mathrm{E}^{n}$ defined above. The cohomology $H^{*}\left(\mathrm{E}^{n} ; \mathbb{C}\right)$ is an exterior algebra, generated by classes $a_{j}$ dual to meridian loops $\gamma_{j}$ about the hyperplanes of $\mathcal{B}^{n}$ (respectively $\mathcal{A}$ ), and one can show that the map $s$ induces a surjection

$$
A\left(\mathcal{B}^{n}\right) \simeq H^{*}\left(\mathrm{E}^{n} ; \mathbb{C}\right) \stackrel{s^{*}}{\longrightarrow} H^{*}(\mathrm{M} ; \mathbb{C}) \simeq A(\mathcal{A})
$$

in (de Rham) cohomology.

Let $\mathcal{B}^{n, \ell}$ be a general position arrangement of $n$ hyperplanes in $\mathbb{C}^{\ell}$, a generic $\ell$-dimensional section of the Boolean arrangement $\mathcal{B}^{n}$ in $\mathbb{C}^{n}$. The Orlik-Solomon algebra $A\left(\mathcal{B}^{n, \ell}\right)$ is the rank $\ell$ truncation of the exterior algebra $A\left(\mathcal{B}^{n}\right)$. We have $A^{q}\left(\mathcal{B}^{n, \ell}\right)=A^{q}\left(\mathcal{B}^{n}\right)$ for $q \leq \ell$ and $A^{q}\left(\mathcal{B}^{n, \ell}\right)=0$ for $q>\ell$. The complement of the arrangement $\mathcal{A}$ in $\mathbb{C}^{\ell}$ has the homotopy type of an $\ell$-dimensional complex, so the cohomology of the complement vanishes in higher dimensions, $H^{q}(\mathrm{M} ; \mathbb{C})=$ $A^{q}(\mathcal{A})=0$ for $q>\ell$. These facts yield the following result.

Proposition 2.2. The map $s: \mathrm{M} \rightarrow \mathrm{E}^{n}$ induces a surjection $s^{*}: A\left(\mathcal{B}^{n, \ell}\right) \rightarrow A(\mathcal{A})$.

A collection of weights $\lambda=\left(\lambda_{1}, \ldots, \lambda_{n}\right) \in \mathbb{C}^{n}$ gives rise to compatible rank one local systems on the complements $\mathrm{E}^{n}, \mathrm{E}^{n, \ell}$, and $\mathrm{M}$ of the arrangements $\mathcal{B}^{n}, \mathcal{B}^{n, \ell}$, and $\mathcal{A}$ as follows. Associated to $\lambda$, there is a rank one representation $\rho: \pi_{1}\left(\mathrm{E}^{n}\right) \rightarrow \mathbb{C}^{*}$ given by $\gamma_{j} \mapsto \exp \left(-2 \pi \mathrm{i} \lambda_{j}\right)$ for any meridian loop $\gamma_{j}$ about the $j$-th hyperplane of $\mathcal{B}^{n}$, and a corresponding rank one local system $\mathcal{L}$ on $\mathrm{E}^{n}$. Let $s: \mathrm{M} \rightarrow \mathrm{E}^{n}$ and $\sigma: \mathrm{E}^{n, \ell} \rightarrow \mathrm{E}^{n}$ be the maps from Proposition 2.1 realizing $\mathrm{M}$ and $\mathrm{E}^{n, \ell}$ as sections of 
$E^{n}$. Via these maps, there are induced local systems on $M$ and $E^{n, \ell}$ corresponding to representations of $\pi_{1}(\mathrm{M})$ and $\pi_{1}\left(\mathrm{E}^{n, \ell}\right)$ defined as above. For brevity, we also use $\mathcal{L}$ to denote these local systems.

One can use stratified Morse theory to construct a cochain complex $K^{\bullet}(\mathcal{A})$, the cohomology of which is naturally isomorphic to the local system cohomology $H^{*}(\mathrm{M} ; \mathcal{L})$, see 3, 田. Briefly, let $\emptyset=\mathcal{F}^{-1} \subset \mathcal{F}^{0} \subset \mathcal{F}^{1} \subset \cdots \subset \mathcal{F}^{\ell}=\mathbb{C}^{\ell}$ be a flag in $\mathbb{C}^{\ell}$ which is transverse to the arrangement $\mathcal{A}$, and let $\mathrm{M}^{q}=\mathcal{F}^{q} \cap \mathrm{M}$ for each $q$. Let $K^{q}=K^{q}(\mathcal{A})=H^{q}\left(\mathrm{M}^{q}, \mathrm{M}^{q-1} ; \mathcal{L}\right)$, and let $\Delta^{q}: H^{q}\left(\mathrm{M}^{q}, \mathrm{M}^{q-1} ; \mathcal{L}\right) \rightarrow$ $H^{q+1}\left(\mathrm{M}^{q+1}, \mathrm{M}^{q} ; \mathcal{L}\right)$ be the boundary homomorphism of the triple $\left(\mathrm{M}^{q+1}, \mathrm{M}^{q}, \mathrm{M}^{q-1}\right)$.

Theorem 2.3 ([3]).

1. For each $q, 0 \leq q \leq \ell$, we have $H^{i}\left(\mathrm{M}^{q}, \mathrm{M}^{q-1} ; \mathcal{L}\right)=0$ if $i \neq q$, and $\operatorname{dim}_{\mathbb{C}} H^{q}\left(\mathrm{M}^{q}, \mathrm{M}^{q-1} ; \mathcal{L}\right)=b_{q}(\mathcal{A})$ is equal to the $q$-th Betti number of $\mathrm{M}$ with trivial local coefficients $\mathbb{C}$.

2. The system of complex vector spaces and linear maps $\left(K^{\bullet}, \Delta^{\bullet}\right)$,

$$
K^{0} \stackrel{\Delta^{0}}{\longrightarrow} K^{1} \stackrel{\Delta^{1}}{\longrightarrow} K^{2} \longrightarrow \cdots \longrightarrow K^{\ell-1} \stackrel{\Delta^{\ell-1}}{\longrightarrow} K^{\ell},
$$

is a complex $\left(\Delta^{q+1} \circ \Delta^{q}=0\right)$. The cohomology of this complex is naturally isomorphic to $H^{*}(\mathrm{M} ; \mathcal{L})$, the cohomology of $\mathrm{M}$ with coefficients in $\mathcal{L}$.

\section{Moduli Spaces}

We now consider families of arrangements with a given combinatorial type. Fix a pair $(\ell, n)$ with $n \geq \ell \geq 1$. We consider (multi-)arrangements of $n$ ordered hyperplanes in $\mathbb{C}^{\ell}$. We assume that the arrangement $\mathcal{A}$ contains $\ell$ linearly independent hyperplanes and that these are the first $\ell$ in the linear order. Let $u_{1}, \ldots, u_{\ell}$ be the coordinates of $\mathbb{C}^{\ell}$. Choose linear polynomials $\alpha_{i}=x_{i, 0}+\sum_{j=1}^{\ell} x_{i, j} u_{j}(i=1, \ldots, n)$ which define the hyperplanes of $\mathcal{A}$. Note that the matrix $\left(x_{i, j}\right), 1 \leq i, j \leq \ell$ is invertible by assumption.

We embed the arrangement in projective space and add the hyperplane at infinity as last in the ordering, $H_{n+1}$. The moduli space of all arrangements combinatorially equivalent to $\mathcal{A}$ may be viewed as the set of matrices

$$
\mathbf{x}=\left(\begin{array}{cccc}
x_{1,0} & x_{1,1} & \cdots & x_{1, \ell} \\
x_{2,0} & x_{2,1} & \cdots & x_{2, \ell} \\
\vdots & \vdots & \ddots & \vdots \\
x_{n, 0} & x_{n, 1} & \cdots & x_{n, \ell} \\
1 & 0 & \cdots & 0
\end{array}\right)
$$

whose rows are elements of $\mathbb{C P}^{\ell}$, and whose $(\ell+1) \times(\ell+1)$ minors satisfy certain dependency conditions, see [18, Prop. 9.2.2]. Given an $\ell+1$ element subset $I$ of $[n+1]:=\{1, \ldots, n, n+1\}$, let $\Delta_{I}=\Delta_{I}(\mathbf{x})$ denote the determinant of the submatrix of $\mathbf{x}$ with rows specified by $I$. Corresponding to each such $\mathbf{x}$, we have an arrangement $\mathcal{A}_{\mathbf{x}}$, with hyperplanes defined by the first $n$ rows of the matrix equation $\mathbf{x} \cdot \tilde{\mathbf{u}}=0$, where $\tilde{\mathbf{u}}=\left(\begin{array}{llll}1 & u_{1} & \cdots & u_{\ell}\end{array}\right)^{\top}$, that is combinatorially equivalent to $\mathcal{A}$. Let $\mathrm{M}_{\mathbf{x}}=M\left(\mathcal{A}_{\mathbf{x}}\right)$ be the complement of $\mathcal{A}_{\mathbf{x}}$.

Let $\mathcal{T}$ denote the combinatorial type of the arrangement $\mathcal{A}$, and let $\mathrm{B}(\mathcal{T})$ denote a smooth, connected component of the moduli space of all arrangements of type $\mathcal{T}$. Let

$$
\mathrm{M}(\mathcal{T})=\left\{(\mathbf{x}, \mathrm{u}) \in\left(\mathbb{C P}^{\ell}\right)^{n} \times \mathbb{C}^{\ell} \mid \mathbf{x} \in \mathrm{B}(\mathcal{T}) \text { and } \mathbf{u} \in \mathrm{M}_{\mathbf{x}}\right\}
$$


and define $\pi_{\mathcal{T}}: \mathrm{M}(\mathcal{T}) \rightarrow \mathrm{B}(\mathcal{T})$ by $\pi_{\mathcal{T}}(\mathrm{x}, \mathrm{u})=\mathrm{x}$. A result of Randell 19 implies that $\pi_{\mathcal{T}}: \mathrm{M}(\mathcal{T}) \rightarrow \mathrm{B}(\mathcal{T})$ is a bundle, with fiber $\pi_{\mathcal{T}}^{-1}(\mathbf{x})=\mathrm{M}_{\mathbf{x}}$, the complement of the arrangement defined by $\mathrm{x} \in \mathrm{B}(\mathcal{T})$.

We now construct a number of spaces and bundles related to the moduli space $\mathrm{B}(\mathcal{T})$. For any combinatorial type $\mathcal{T}$, let $\operatorname{ind}(\mathcal{T})$ denote the set of all $\ell+1$ element subsets $I=\left\{i_{1}, \ldots, i_{\ell+1}\right\}$ of $[n+1]$ for which $\Delta_{I} \neq 0$ in type $\mathcal{T}$. If $\mathcal{T}$ is realizable, $\operatorname{ind}(\mathcal{T})$ is the set of all subsets $I$ for which $\left\{H_{i_{1}}, \ldots, H_{i_{\ell+1}}\right\}$ is linearly independent in the projective closure of an arrangement $\mathcal{A}$ of type $\mathcal{T}$. Similarly, let $\operatorname{dep}(\mathcal{T})$ be the set of all $\ell+1$ element subsets $J$ of $[n+1]$ for which $\Delta_{J}=0$ in type $\mathcal{T}$.

Given a type $\mathcal{T}$, let

$$
\mathrm{Y}(\mathcal{T})=\left\{\mathbf{x} \in\left(\mathbb{C P}^{\ell}\right)^{n} \mid \Delta_{I}(\mathbf{x}) \neq 0 \text { for } I \in \operatorname{ind}(\mathcal{T})\right\} .
$$

Then the moduli space $\mathrm{B}(\mathcal{T})$ may be realized as

$$
\mathrm{B}(\mathcal{T})=\left\{\mathbf{x} \in \mathrm{Y}(\mathcal{T}) \mid \Delta_{J}(\mathbf{x})=0 \text { for } J \in \operatorname{dep}(\mathcal{T})\right\} .
$$

Note that if $\mathcal{G}$ is the type of a general position arrangement, then $\operatorname{dep}(\mathcal{G})=\emptyset$, so $\mathrm{B}(\mathcal{G})=\mathrm{Y}(\mathcal{G})$. For any type $\mathcal{T}$, the moduli space $\mathrm{B}(\mathcal{G})$ of all general position arrangements may be realized as

$$
\mathrm{B}(\mathcal{G})=\left\{\mathrm{x} \in \mathrm{Y}(\mathcal{T}) \mid \Delta_{J}(\mathbf{x}) \neq 0 \text { for } J \in \operatorname{dep}(\mathcal{T})\right\} .
$$

If $\mathcal{T} \neq \mathcal{G}$, then $\mathrm{B}(\mathcal{T})$ and $\mathrm{B}(\mathcal{G})$ are disjoint subspaces of $\mathrm{Y}(\mathcal{T})$. Let $i_{\mathcal{T}}: \mathrm{B}(\mathcal{G}) \rightarrow \mathrm{Y}(\mathcal{T})$ and $j_{\mathcal{T}}: \mathrm{B}(\mathcal{T}) \rightarrow \mathrm{Y}(\mathcal{T})$ denote the natural inclusions.

Let $I_{0}=\{1, \ldots, \ell, n+1\}$, and define $\mathcal{T}_{0}$ by $\operatorname{ind}\left(\mathcal{T}_{0}\right)=\left\{I_{0}\right\}$. Then $\mathrm{B}\left(\mathcal{T}_{0}\right)$ is the moduli space of all multi-arrangements with $\left\{H_{1}, \ldots, H_{\ell}, H_{n+1}\right\}$ linearly independent. Given $\mathrm{x} \in \mathrm{B}\left(\mathcal{T}_{0}\right)$, let $\mathcal{B}_{\mathrm{x}}^{n}$ denote the Boolean arrangement of $n$ hyperplanes in $\mathbb{C}^{n}$ with defining polynomial $Q\left(\mathcal{B}_{\mathrm{x}}^{n}\right)$ given by

$$
Q\left(\mathcal{B}_{\mathbf{x}}^{n}\right)=\prod_{i=1}^{\ell}\left(x_{i, 0}+x_{i, 1} u_{1}+\cdots+x_{i, \ell} u_{\ell}\right) \prod_{i=\ell+1}^{n} u_{i},
$$

where $u_{1}, \ldots, u_{\ell}, u_{\ell+1}, \ldots, u_{n}$ are the coordinates for $\mathbb{C}^{n}$. Let $\mathrm{E}_{\mathbf{x}}^{n}=\mathbb{C}^{n} \backslash Q\left(\mathcal{B}_{\mathbf{x}}^{n}\right)^{-1}(0)$ be the complement of the arrangement $\mathcal{B}_{\mathbf{x}}^{n}$. Note that $E_{\mathbf{x}}^{n} \cong\left(\mathbb{C}^{*}\right)^{n}$ is a complex $n$-torus. Define

$$
\mathrm{E}\left(\mathcal{T}_{0}\right)=\left\{(\mathbf{x}, \mathrm{u}) \in\left(\mathbb{C P}^{\ell}\right)^{n} \times \mathbb{C}^{n} \mid \mathbf{x} \in \mathrm{Y}\left(\mathcal{T}_{0}\right) \text { and } \mathrm{u} \in \mathrm{E}_{\mathbf{x}}^{n}\right\}
$$

Proposition 3.1. The natural map $p_{\mathcal{T}_{0}}: \mathrm{E}\left(\mathcal{T}_{0}\right) \rightarrow \mathrm{Y}\left(\mathcal{T}_{0}\right)$ defined by $p_{\mathcal{T}_{0}}(\mathbf{x}, \mathbf{u})=\mathbf{x}$ is a fiber bundle projection, with fiber the complex n-torus $p_{\mathcal{T}_{0}}^{-1}(\mathbf{x})=\mathrm{E}_{\mathbf{x}}^{n}$.

Proof. This may be established using the Thom Isotopy Lemma by modifying the argument given by Randell [19].

For each type $\mathcal{T}$ for which $I_{0} \in \operatorname{ind}(\mathcal{T})$, let $k_{\mathcal{T}}: \mathrm{Y}(\mathcal{T}) \rightarrow \mathrm{Y}\left(\mathcal{T}_{0}\right)$ denote the natural inclusion. Recall that $j_{\mathcal{T}}: \mathrm{B}(\mathcal{T}) \rightarrow \mathrm{Y}(\mathcal{T})$ denotes the inclusion of the moduli space of $\mathcal{T}$ in $Y(\mathcal{T})$. By pulling back the above bundle along the inclusion maps $k_{\mathcal{T}}: \mathrm{Y}(\mathcal{T}) \rightarrow \mathrm{Y}\left(\mathcal{T}_{0}\right)$ and $k_{\mathcal{T}} \circ j_{\mathcal{T}}: \mathrm{B}(\mathcal{T}) \rightarrow \mathrm{Y}\left(\mathcal{T}_{0}\right)$, we obtain bundles over $\mathrm{Y}(\mathcal{T})$ and $\mathrm{B}(\mathcal{T})$, with fiber the complex $n$-torus.

Denote these bundles by

$$
p_{\mathcal{T}}: \mathrm{E}(\mathcal{T}) \rightarrow \mathrm{Y}(\mathcal{T}) \quad \text { and } \quad p_{\mathcal{T}}^{\prime}: \mathrm{E}^{\prime}(\mathcal{T}) \rightarrow \mathrm{B}(\mathcal{T})
$$


respectively. The total spaces of these bundles may be realized as

$$
\begin{aligned}
\mathrm{E}(\mathcal{T}) & =\left\{(\mathbf{x}, \mathrm{u}) \in\left(\mathbb{C P}^{\ell}\right)^{n} \times \mathbb{C}^{n} \mid \mathbf{x} \in \mathrm{Y}(\mathcal{T}) \text { and } \mathrm{u} \in \mathrm{E}_{\mathbf{x}}^{n}\right\} \text { and } \\
\mathrm{E}^{\prime}(\mathcal{T}) & =\left\{(\mathbf{x}, \mathrm{u}) \in\left(\mathbb{C P}^{\ell}\right)^{n} \times \mathbb{C}^{n} \mid \mathbf{x} \in \mathrm{B}(\mathcal{T}) \text { and } \mathrm{u} \in \mathrm{E}_{\mathbf{x}}^{n}\right\} .
\end{aligned}
$$

The bundle $p_{\mathcal{T}}^{\prime}: \mathrm{E}^{\prime}(\mathcal{T}) \rightarrow \mathrm{B}(\mathcal{T})$ and the moduli space bundle $\pi_{\mathcal{T}}: \mathrm{M}(\mathcal{T}) \rightarrow \mathrm{B}(\mathcal{T})$ are related as follows.

Proposition 3.2. There is a bundle map $S: \mathrm{M}(\mathcal{T}) \rightarrow \mathrm{E}^{\prime}(\mathcal{T})$ covering the identity map of $\mathrm{B}(\mathcal{T})$.

Proof. Let $\mathrm{x} \in \mathrm{B}(\mathcal{T})$. Corresponding to $\mathrm{x}$, we have an arrangement $\mathcal{A}_{\mathbf{x}}$ of type $\mathcal{T}$ in $\mathbb{C}^{\ell}$, and a Boolean arrangement $\mathcal{B}_{\mathbf{x}}^{n}$ in $\mathbb{C}^{n}$. These arrangements have defining polynomials

$$
\begin{aligned}
Q\left(\mathcal{A}_{\mathbf{x}}\right) & =\prod_{i=1}^{\ell}\left(x_{i, 0}+x_{i, 1} u_{1}+\cdots+x_{i, \ell} u_{\ell}\right) \prod_{i=\ell+1}^{n}\left(x_{i, 0}+x_{i, 1} u_{1}+\cdots+x_{i, \ell} u_{\ell}\right) \text { and } \\
Q\left(\mathcal{B}_{\mathbf{x}}^{n}\right) & =\prod_{i=1}^{\ell}\left(x_{i, 0}+x_{i, 1} u_{1}+\cdots+x_{i, \ell} u_{\ell}\right) \prod_{i=\ell+1}^{n} u_{i}
\end{aligned}
$$

where $u_{1}, \ldots, u_{\ell}$ are coordinates for $\mathbb{C}^{\ell}$ and $u_{1}, \ldots, u_{n}$ are coordinates for $\mathbb{C}^{n}$. It follows that the map $\mathbb{C}^{\ell} \rightarrow \mathbb{C}^{n},\left(u_{1}, \ldots, u_{\ell}\right) \mapsto\left(u_{1}, \ldots, u_{\ell}, \alpha_{\ell+1}, \ldots, \alpha_{n}\right)$, defined in (2.1) restricts to a map $s_{\mathrm{x}}: \mathrm{M}_{\mathrm{x}} \rightarrow \mathrm{E}_{\mathrm{x}}^{n}$ of the complement of $\mathcal{A}_{\mathrm{x}}$ to the complement of $\mathcal{B}_{\mathbf{x}}^{n}$, see Proposition 2.1. Defining $S: \mathrm{M}(\mathcal{T}) \rightarrow \mathrm{E}^{\prime}(\mathcal{T})$ by $S(\mathbf{x}, \mathrm{u})=\left(\mathbf{x}, s_{\mathbf{x}}(\mathrm{u})\right)$ yields the desired bundle map.

\section{Representations}

Let $\mathcal{A}$ be an essential arrangement of $n$ hyperplanes in $\mathbb{C}^{\ell}$ of combinatorial type $\mathcal{T}$, and let $\mathrm{B}(\mathcal{T})$ be a smooth, connected component of the corresponding moduli space. For each $\mathrm{x} \in \mathrm{B}(\mathcal{T})$, a collection of complex weights $\lambda=\left(\lambda_{1}, \ldots, \lambda_{n}\right)$ determines a rank one local system $\mathcal{L}_{\mathbf{x}}$ on the complement $\mathrm{M}_{\mathbf{x}}$ of the arrangement $\mathcal{A}_{\mathbf{x}}$, with monodromy $\exp \left(-2 \pi \mathrm{i} \lambda_{j}\right)$ about the hyperplane $H_{j} \in \mathcal{A}_{\mathbf{x}}$.

Since the fiber bundle $\pi: \mathrm{M}(\mathcal{T}) \rightarrow \mathrm{B}(\mathcal{T})$ is locally trivial, there is an associated vector bundle $\pi^{q}: \mathbf{H}^{q} \rightarrow \mathrm{B}(\mathcal{T})$, with fiber $\left(\pi^{q}\right)^{-1}(\mathbf{x})=H^{q}\left(\mathrm{M}_{\mathbf{x}} ; \mathcal{L}_{\mathbf{x}}\right)$ at $\mathbf{x} \in \mathrm{B}(\mathcal{T})$ for each $q, 0 \leq q \leq \ell$. The transition functions of this vector bundle are locally constant. Fixing a basepoint $\mathrm{x} \in \mathrm{B}(\mathcal{T})$, the operation of parallel translation of fibers over curves in $\mathrm{B}(\mathcal{T})$ in the vector bundle $\pi^{q}: \mathbf{H}^{q} \rightarrow \mathrm{B}(\mathcal{T})$ provides a complex representation

$$
\Psi^{q}: \pi_{1}(\mathrm{~B}(\mathcal{T}), \mathbf{x}) \longrightarrow \operatorname{Aut}_{\mathbb{C}}\left(H^{q}\left(\mathrm{M}_{\mathbf{x}} ; \mathcal{L}_{\mathbf{x}}\right)\right) .
$$

Write $\Psi^{q}=\Psi_{\mathcal{T}}^{q}$ to indicate the dependence of this representation on the type $\mathcal{T}$.

By Theorem 2.3, the local system cohomology of $M_{\mathbf{x}}$ may be computed using the Morse theoretic complex $K^{\bullet}\left(\mathcal{A}_{\mathbf{x}}\right)$. The fundmental group of $\mathrm{B}(\mathcal{T})$ acts by chain automorphisms on this complex, see [6. Cor. 3.2], yielding a representation

$$
\Phi^{\bullet}: \pi_{1}(\mathrm{~B}(\mathcal{T}), \mathbf{x}) \longrightarrow \operatorname{Aut}_{\mathbb{C}}\left(K^{\bullet}\left(\mathcal{A}_{\mathbf{x}}\right)\right) .
$$

As above, write $\Phi^{\bullet}=\Phi_{\mathcal{T}}^{\bullet}$ to indicate the dependence of this representation on the combinatorial type $\mathcal{T}$.

Theorem $4.1([6])$. The representation $\Psi_{\mathcal{T}}^{q}: \pi_{1}(\mathrm{~B}(\mathcal{T}), \mathbf{x}) \rightarrow \operatorname{Aut}_{\mathbb{C}}\left(H^{q}\left(\mathrm{M}_{\mathbf{x}} ; \mathcal{L}_{\mathbf{x}}\right)\right)$ is induced by the representation $\Phi_{\mathcal{T}}^{\bullet}: \pi_{1}(\mathrm{~B}(\mathcal{T}), \mathbf{x}) \rightarrow \operatorname{Aut}_{\mathbb{C}}\left(K^{\bullet}\left(\mathcal{A}_{\mathbf{x}}\right)\right)$. 
The constructions of the previous section provide additional representations of the fundamental group of $\mathrm{B}(\mathcal{T})$. Recall that $I_{0}=\{1, \ldots, \ell, n+1\}$, and assume $I_{0} \in$ $\operatorname{ind}(\mathcal{T})$. Then there is a bundle $p_{\mathcal{T}}^{\prime}: \mathrm{E}^{\prime}(\mathcal{T}) \rightarrow \mathrm{B}(\mathcal{T})$, with fiber $\mathrm{E}_{\mathbf{x}}^{n}$, the complement of the Boolean arrangement $\mathcal{B}_{\mathbf{x}}^{n}$ in $\mathbb{C}^{n}$. Recall also that the the weights $\lambda$ give rise to a local system on $\mathrm{E}_{\mathrm{x}}^{n}$, which we also denote by $\mathcal{L}_{\mathbf{x}}$ since it is compatible with the local system on $M_{\mathbf{x}}$. As above, the fundamental group of $B(\mathcal{T})$ acts by chain automorphisms on the cochain complex $K^{\bullet}\left(\mathcal{B}_{\mathbf{x}}^{n}\right)$ associated with this arrangement, yielding a representation

$$
\widetilde{\Phi}_{\mathcal{T}}^{\bullet}: \pi_{1}(\mathrm{~B}(\mathcal{T}), \mathbf{x}) \longrightarrow \operatorname{Aut}_{\mathbb{C}}\left(K^{\bullet}\left(\mathcal{B}_{\mathbf{x}}^{n}\right)\right)
$$

By Proposition 3.2, there is a bundle map $s: \mathrm{M}(\mathcal{T}) \rightarrow \mathrm{E}^{\prime}(\mathcal{T})$, which restricts to $s_{\mathbf{x}}: \mathrm{M}_{\mathbf{x}} \rightarrow \mathrm{E}_{\mathbf{x}}^{n}$ on fibers. The map $s_{\mathbf{x}}$ induces a chain map $s_{\mathbf{x}}^{\bullet}: K^{\bullet}\left(\mathcal{B}_{\mathbf{x}}^{n}\right) \rightarrow K^{\bullet}\left(\mathcal{A}_{\mathbf{x}}\right)$, see [ [4 Prop. 2.11], which is compatible with the representations $\Phi_{\mathcal{T}}^{\bullet}$ and $\widetilde{\Phi}_{\mathcal{T}}^{\bullet}$ of (4.2) and (4.3) in the following sense.

Proposition 4.2. For each $\gamma \in \pi_{1}(\mathrm{~B}(\mathcal{T}), \mathbf{x})$, there is a commutative diagram

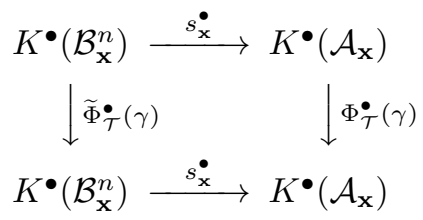

Remark 4.3. Recall that $\mathcal{G}$ denotes the combinatorial type of a general position arrangement of $n$ hyperplanes in $\mathbb{C}^{\ell}$. If $\mathbf{y} \in \mathrm{B}(\mathcal{G})$ is a point in the moduli space of all such arrangements, let $\mathcal{B}_{\mathbf{y}}^{n, \ell}$ denote the corresponding arrangement. The above discussion, when applied to the arrangement $\mathcal{B}_{\mathbf{y}}^{n, \ell}$, yields the following commutative diagram

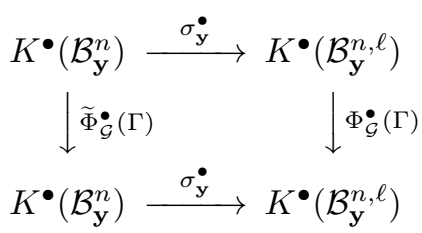

where $\Gamma \in \pi_{1}(\mathrm{~B}(\mathcal{G}), \mathbf{y})$ and $\sigma_{\mathbf{y}}^{\bullet}: K^{\bullet}\left(\mathcal{B}_{\mathbf{y}}^{n}\right) \rightarrow K^{\bullet}\left(\mathcal{B}_{\mathbf{y}}^{n, \ell}\right)$ is the chain map induced by the bundle map $\sigma: \mathrm{M}(\mathcal{G}) \rightarrow \mathrm{E}^{\prime}(\mathcal{G})$ of Proposition 3.2 in this particular case.

The complement $\mathrm{E}_{\mathbf{y}}^{n, \ell}=M\left(\mathcal{B}_{\mathbf{y}}^{n, \ell}\right)$ of the general position arrangement $\mathcal{B}_{\mathbf{y}}^{n, \ell}$ may be realized as a generic $\ell$-dimensional section of the complement $\mathrm{E}_{\mathbf{y}}^{n}=M\left(\mathcal{B}_{\mathbf{y}}^{n}\right)$ of the Boolean arrangement $\mathcal{B}_{\mathbf{y}}^{n}$, and the map $\sigma_{\mathbf{y}}: \mathrm{E}_{\mathbf{y}}^{n, \ell} \rightarrow \mathrm{E}_{\mathbf{y}}^{n}$ as the inclusion of this generic section. In the notation established in the construction of the Morse theoretic complex $K^{\bullet}$ preceding Theorem 2.3, we have $\mathrm{E}_{\mathbf{y}}^{n, \ell}=\mathcal{F}^{\ell} \cap \mathrm{E}_{\mathbf{y}}^{n}$. From this construction, it is clear that the complex $K^{\bullet}\left(\mathcal{B}_{\mathbf{y}}^{n, \ell}\right)$ is the rank $\ell$ truncation of the complex $K^{\bullet}\left(\mathcal{B}_{\mathbf{y}}^{n}\right)$ :

$$
K^{q}\left(\mathcal{B}_{\mathbf{y}}^{n, \ell}\right)=\left\{\begin{array}{ll}
K^{q}\left(\mathcal{B}_{\mathbf{y}}^{n}\right) & \text { if } q \leq \ell, \\
0 & \text { if } q>\ell,
\end{array} \quad \text { and } \quad \sigma_{\mathbf{y}}^{q}= \begin{cases}\text { id }: K^{q}\left(\mathcal{B}_{\mathbf{y}}^{n}\right) \rightarrow K^{q}\left(\mathcal{B}_{\mathbf{y}}^{n}\right) & \text { if } q \leq \ell \\
0 & \text { if } q>\ell\end{cases}\right.
$$

See [3, §7] and [4, Ex. 2.7] for explicit constructions of these complexes.

The following is a consequence of the above discussion. 
Proposition 4.4. The representations $\Phi_{\mathcal{G}}^{\bullet}: \pi_{1}(\mathrm{~B}(\mathcal{G}), \mathbf{y}) \rightarrow \operatorname{Aut}_{\mathbb{C}}\left(K^{\bullet}\left(\mathcal{B}_{\mathbf{y}}^{n, \ell}\right)\right)$ and $\widetilde{\Phi}_{\mathcal{G}}^{\bullet}: \pi_{1}(\mathrm{~B}(\mathcal{G}), \mathbf{y}) \rightarrow \operatorname{Aut}_{\mathbb{C}}\left(K^{\bullet}\left(\mathcal{B}_{\mathbf{y}}^{n}\right)\right)$ satisfy $\Phi_{\mathcal{G}}^{q}=\widetilde{\Phi}_{\mathcal{G}}^{q}$ for $q \leq \ell$.

Now recall the bundle $p_{\mathcal{T}}: \mathrm{E}(\mathcal{T}) \rightarrow \mathrm{Y}(\mathcal{T})$, with fiber $\mathrm{E}_{\mathbf{x}}^{n}=M\left(\mathcal{B}_{\mathbf{x}}^{n}\right)$, and the inclusion map $j_{\mathcal{T}}: \mathrm{B}(\mathcal{T}) \rightarrow \mathrm{Y}(\mathcal{T})$ from Section 3. As discussed above, an element $\gamma \in \pi_{1}(\mathrm{~B}(\mathcal{T}), \mathbf{x})$ induces a chain automorphism $\widetilde{\Phi}_{\mathcal{T}}^{\bullet}(\gamma)$ of the cochain complex $K^{\bullet}\left(\mathcal{B}_{\mathrm{x}}^{n}\right)$. Let $g: \mathbb{S}^{1} \rightarrow \mathrm{B}(\mathcal{T})$ be a loop representing $\gamma$. Then $j_{\mathcal{T}} \circ g: \mathbb{S}^{1} \rightarrow \mathrm{Y}(\mathcal{T})$ is a loop representing an element, say $\xi \in \pi_{1}(\mathrm{Y}(\mathcal{T}), \mathbf{x})$, and $\xi$ also induces a chain automorphism of the complex $K^{\bullet}\left(\mathcal{B}_{\mathbf{x}}^{n}\right)$, which we denote by $\Xi^{\bullet}(\gamma)$.

Lemma 4.5. The chain automorphisms $\widetilde{\Phi}_{\mathcal{T}}^{\bullet}(\gamma)$ and $\Xi^{\bullet}(\gamma)$ of the complex $K^{\bullet}\left(\mathcal{B}_{\mathbf{x}}^{n}\right)$ coincide, $\widetilde{\Phi}_{\mathcal{T}}^{\bullet}(\gamma)=\Xi^{\bullet}(\gamma)$.

Proof. Let $g^{*}\left(p_{\mathcal{T}}^{\prime}\right)$ denote the pullback of the bundle $p_{\mathcal{T}}^{\prime}: \mathrm{E}^{\prime}(\mathcal{T}) \rightarrow \mathrm{B}(\mathcal{T})$ along the map $g: \mathbb{S}^{1} \rightarrow \mathrm{B}(\mathcal{T})$, and let $\left(j_{\mathcal{T}} \circ g\right)^{*}\left(p_{\mathcal{T}}\right)$ be the pullback of $p_{\mathcal{T}}: \mathrm{E}(\mathcal{T}) \rightarrow \mathrm{Y}(\mathcal{T})$ along $j_{\mathcal{T}} \circ g: \mathbb{S}^{1} \rightarrow \mathrm{Y}(\mathcal{T})$. The chain automorphisms $\widetilde{\Phi}_{\mathcal{T}}^{\bullet}(\gamma)$ and $\Xi^{\bullet}(\gamma)$ are induced by the monodromies of the bundles $g^{*}\left(p_{\mathcal{T}}^{\prime}\right)$ and $\left(j_{\mathcal{T}} \circ g\right)^{*}\left(p_{\mathcal{T}}\right)$ respectively. Checking that these two bundles are identical, we conclude that the two monodromies are the same. The result follows.

For any combinatorial type $\mathcal{T}$, there is an inclusion $i_{\mathcal{T}}: \mathrm{B}(\mathcal{G}) \rightarrow \mathrm{Y}(\mathcal{T})$ of the moduli space $\mathrm{B}(\mathcal{G})=\mathrm{Y}(\mathcal{G})$ of all general position arrangements in the space $\mathrm{Y}(\mathcal{T})$. More generally, if $\mathcal{S}$ and $\mathcal{T}$ are types satisfying $\operatorname{ind}(\mathcal{T}) \subseteq \operatorname{ind}(\mathcal{S})$, there is an inclusion $i_{\mathcal{S}, \mathcal{T}}: \mathrm{Y}(\mathcal{S}) \rightarrow \mathrm{Y}(\mathcal{T})$

Proposition 4.6. If $\mathcal{S}$ and $\mathcal{T}$ are combinatorial types with $\operatorname{ind}(\mathcal{T}) \subseteq \operatorname{ind}(\mathcal{S})$, the map $\left(i_{\mathcal{S}, \mathcal{T}}\right)_{*}: H_{1}(\mathrm{Y}(\mathcal{S})) \rightarrow H_{1}(\mathrm{Y}(\mathcal{T}))$ induced by the inclusion $i_{\mathcal{S}, \mathcal{T}}: \mathrm{Y}(\mathcal{S}) \rightarrow \mathrm{Y}(\mathcal{T})$ is surjective.

Proof. It suffices to consider the case where $\operatorname{ind}(\mathcal{S})=\operatorname{ind}(\mathcal{T}) \cup\{J\}$ for some $J=$ $\left\{j_{1}, \ldots, j_{\ell+1}\right\} \subset[n+1]$ with $J \notin \operatorname{ind}(\mathcal{T})$. Let $\mathcal{T}_{J}$ be the type with $\operatorname{ind}\left(\mathcal{T}_{J}\right)=\{J\}$, and let $U=\mathrm{Y}(\mathcal{T})$ and $V=\mathrm{Y}\left(\mathcal{T}_{J}\right)=\left(\mathbb{C P}^{\ell}\right)^{n} \backslash\left\{\Delta_{J}=0\right\}$. Then $U \cap V=\mathrm{Y}(\mathcal{S})$, and $U \cup V=\left(\mathbb{C P}^{\ell}\right)^{n} \backslash \bigcup_{q=1}^{k}\left(\left\{\Delta_{I_{q}}=0\right\} \cap\left\{\Delta_{J}=0\right\}\right)$, where $\operatorname{ind}(\mathcal{T})=\left\{I_{1}, \ldots, I_{k}\right\}$. Since $\left\{\Delta_{I_{q}}=0\right\} \cap\left\{\Delta_{J}=0\right\}$ is of complex codimension two in $\left(\mathbb{C P}^{\ell}\right)^{n}$ for each $q$, the inclusion of $U \cup V$ in $\left(\mathbb{C P}^{\ell}\right)^{n}$ induces an isomorphism $H_{1}(U \cup V) \stackrel{\sim}{\rightarrow} H_{1}\left(\left(\mathbb{C P}^{\ell}\right)^{n}\right)$. Thus $H_{1}(U \cup V)=0$. With these observations, the Mayer-Vietoris sequence in homology is of the form

$$
\cdots \rightarrow H_{2}(U \cup V) \rightarrow H_{1}(\mathrm{Y}(\mathcal{S})) \rightarrow H_{1}(\mathrm{Y}(\mathcal{T})) \oplus H_{1}\left(\mathrm{Y}\left(\mathcal{T}_{J}\right)\right) \rightarrow H_{1}(U \cup V)=0 .
$$

Since the map $H_{1}(\mathrm{Y}(\mathcal{S})) \rightarrow H_{1}(\mathrm{Y}(\mathcal{T})) \oplus H_{1}\left(\mathrm{Y}\left(\mathcal{T}_{J}\right)\right)$ is given by $\left(\left(i_{\mathcal{S}, \mathcal{T}}\right)_{*},\left(i_{\mathcal{S}, \mathcal{T}_{J}}\right)_{*}\right)$, the result follows.

Corollary 4.7. For any combinatorial type $\mathcal{T}$, the inclusion $i_{\mathcal{T}}: B(\mathcal{G}) \rightarrow \mathrm{Y}(\mathcal{T})$ induces a surjection $\left(i_{\mathcal{T}}\right)_{*}: H_{1}(\mathrm{~B}(\mathcal{G})) \rightarrow H_{1}(\mathrm{Y}(\mathcal{T}))$.

The above results provide a means for comparing the representations

$$
\Phi_{\mathcal{T}}^{\bullet}: \pi_{1}(\mathrm{~B}(\mathcal{T}), \mathbf{x}) \rightarrow \operatorname{Aut}_{\mathbb{C}}\left(K^{\bullet}\left(\mathcal{A}_{\mathbf{x}}\right)\right) \quad \text { and } \quad \Phi_{\mathcal{G}}^{\bullet}: \pi_{1}(\mathrm{~B}(\mathcal{G}), \mathbf{y}) \rightarrow \operatorname{Aut}_{C}\left(K^{\bullet}\left(\mathcal{B}_{\mathbf{y}}^{n, \ell}\right),\right.
$$

corresponding to an arbitrary arrangement of $n$ hyperplanes in $\mathbb{C}^{\ell}$ and a general position arrangement of $n$ hyperplanes in $\mathbb{C}^{\ell}$. 
Theorem 4.8. Let $\mathcal{T}$ be a combinatorial type with $I_{0} \in \operatorname{ind}(\mathcal{T})$. Let $\mathcal{A}_{\mathbf{x}}$ be an arrangement of type $\mathcal{T}$, corresponding to a point $\mathrm{x} \in \mathrm{B}(\mathcal{T})$. Let $\mathcal{B}_{\mathbf{y}}^{n, \ell}$ be a general position arrangement, corresponding to a point $\mathbf{y} \in \mathrm{B}(\mathcal{G})$. Then for any element $\gamma \in \pi_{1}(\mathrm{~B}(\mathcal{T}), \mathbf{x})$, there is an element $\Gamma \in \pi_{1}(\mathrm{~B}(\mathcal{G}), \mathbf{y})$ so that the diagram

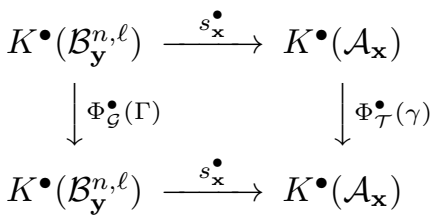

commutes up to conjugacy.

Proof. Let $g: \mathbb{S}^{1} \rightarrow \mathrm{B}(\mathcal{T})$ be a loop representing $\gamma \in \pi_{1}(\mathrm{~B}(\mathcal{T}), \mathbf{x})$. Then $j_{\mathcal{T}} \circ g$ : $\mathbb{S}^{1} \rightarrow \mathrm{B}(\mathcal{T}) \rightarrow \mathrm{Y}(\mathcal{T})$ is a loop representing $\xi \in \pi_{1}(\mathrm{Y}(\mathcal{T}), \mathrm{x})$. Using Proposition 4.2 and Lemma 1.5 , we have the commutative diagram

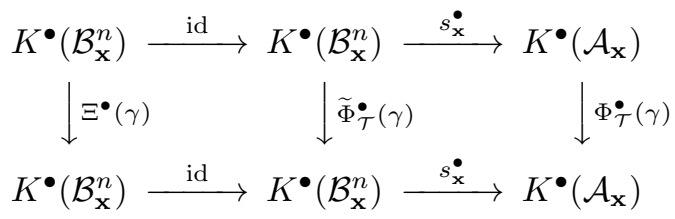

where $\Xi^{\bullet}(\gamma)$ is the chain automorphism induced by $\xi$.

Denote the homology class of $\xi$ in $H_{1}(\mathrm{Y}(\mathcal{T}))$ by $[\xi]$. By Corollary 4.7, the inclusion $i_{\mathcal{T}}: \mathrm{B}(\mathcal{G}) \rightarrow \mathrm{Y}(\mathcal{T})$ induces a surjection $\left(i_{\mathcal{T}}\right)_{*}: H_{1}(\mathrm{~B}(\mathcal{G})) \rightarrow H_{1}(\mathrm{Y}(\mathcal{T}))$. Let $\mathbf{y} \in \mathrm{B}(\mathcal{G})$ be a basepoint (the point $\mathbf{x} \in \mathrm{B}(\mathcal{T})$ is not in $\mathrm{B}(\mathcal{G})$ if $\mathcal{T} \neq \mathcal{G})$. Let $\Gamma \in$ $\pi_{1}(\mathrm{~B}(\mathcal{G}), \mathbf{y})$ be an element whose homology class $[\Gamma]$ satisfies $\left(i_{\mathcal{T}}\right)_{*}([\Gamma])=[\xi]$. Then the chain automorphisms induced by $\left(i_{\mathcal{T}}\right)_{\#}(\Gamma) \in \pi_{1}(\mathrm{Y}(\mathcal{T}), \mathbf{y})$ and $\xi \in \pi_{1}(\mathrm{Y}(\mathcal{T}), \mathbf{x})$ are conjugate, so we have the commutative diagram

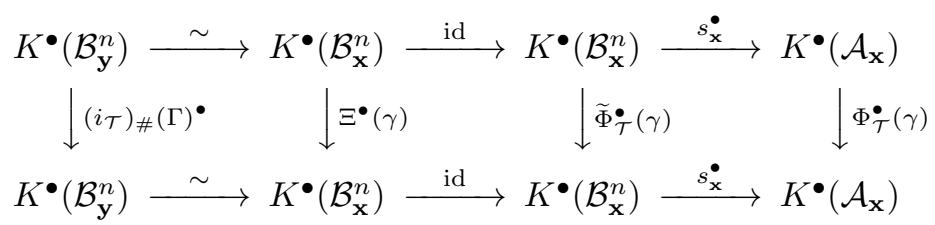

Recall that $\mathrm{B}(\mathcal{G})=\mathrm{Y}(\mathcal{G})$. Over $\mathrm{B}(\mathcal{G})$ and $\mathrm{Y}(\mathcal{T})$, we have bundles $p_{\mathcal{G}}: \mathrm{E}(\mathcal{G}) \rightarrow$ $\mathrm{B}(\mathcal{G})$ and $p_{\mathcal{T}}: \mathrm{E}(\mathcal{T}) \rightarrow \mathrm{Y}(\mathcal{T})$, each with fiber $\mathrm{E}_{\mathbf{y}}^{n}$, the complement of the Boolean arrangement $\mathcal{B}_{\mathbf{y}}^{n}$. Let $G: \mathbb{S}^{1} \rightarrow \mathrm{B}(\mathcal{G})$ be a loop representing $\Gamma \in \pi_{1}(\mathrm{~B}(\mathcal{G}), \mathbf{y})$. It is then readily checked that the pullbacks $G^{*}\left(p_{\mathcal{G}}\right)$ and $\left(i_{\mathcal{T}} \circ G\right)^{*}\left(p_{\mathcal{T}}\right)$ of the two bundles above are identical. Consequently, the chain automorphisms $\widetilde{\Phi}_{\mathcal{G}}^{\bullet}(\Gamma)$ and $\left(i_{\mathcal{T}}\right)_{\#}(\Gamma)^{\bullet}$ of $K^{\bullet}\left(\mathcal{B}_{\mathbf{y}}^{n}\right)$ induced by $\Gamma$ and $\left(i_{\mathcal{T}}\right)_{\#}(\Gamma)$ are equal. This fact, together with (4.4), yields the commutative diagram

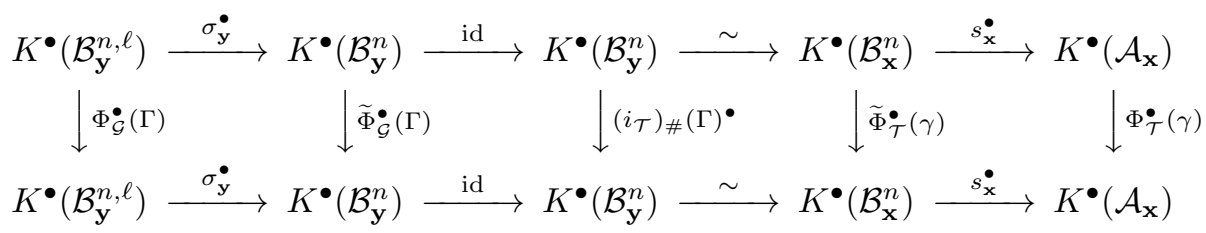

Since $\sigma_{\mathbf{y}}^{q}=\operatorname{id~}: K^{q}\left(\mathcal{B}_{\mathbf{y}}^{n, \ell}\right) \stackrel{=}{\rightarrow} K^{q}\left(\mathcal{B}^{n}, \mathbf{y}\right)$ and $\Phi_{\mathcal{G}}^{\bullet}(\Gamma)=\widetilde{\Phi}_{\mathcal{G}}^{\bullet}(\Gamma)$ for $q \leq \ell$ by Remark 4.3 and Proposition 4.4, and $K^{q}\left(\mathcal{B}_{\mathbf{y}}^{n, \ell}\right)=K^{q}\left(\mathcal{A}_{\mathbf{x}}\right)=0$ for $q>\ell$, this completes the proof. 
Given $\gamma \in \pi_{1}(\mathrm{~B}(\mathcal{T}), \mathbf{x})$, we say that $\Gamma \in \pi_{1}(\mathrm{~B}(\mathcal{G}), \mathbf{y})$ is compatible with $\gamma$ if their homology classes satisfy $\left(i_{\mathcal{T}}\right)_{*}([\Gamma])=\left(j_{\mathcal{T}}\right)_{*}([\gamma])$. The results established above show that for every $\gamma$ there exists a compatible $\Gamma$. A key tool in this paper is the relationship between the automorphism $\Phi_{\mathcal{T}}^{\bullet}(\gamma) \in \operatorname{Aut}_{C} K^{\bullet}\left(\mathcal{A}_{\mathbf{x}}\right)$ for an arbitrary arrangement and the automorphism $\Phi_{\mathcal{C}}^{\bullet}(\Gamma) \in \operatorname{Aut}_{\mathbb{C}} K^{\bullet}\left(\mathcal{B}_{\mathbf{y}}^{n, \ell}\right)$ for a general position arrangement provided by Theorem 4.8 for compatible $\gamma$ and $\Gamma$. This relationship extends to cohomology. By Theorem 4.1, the representation $\Psi_{\mathcal{T}}^{q}$ : $\pi_{1}(\mathrm{~B}(\mathcal{T}), \mathbf{x}) \rightarrow \operatorname{Aut}_{\mathbb{C}}\left(H^{q}\left(\mathrm{M}_{\mathbf{x}} ; \mathcal{L}_{\mathbf{x}}\right)\right)$ in cohomology is induced by the representation $\Phi_{\mathcal{T}}^{\bullet}: \pi_{1}(\mathrm{~B}(\mathcal{T}), \mathbf{x}) \rightarrow \operatorname{Aut}_{\mathbb{C}}\left(K^{\bullet}\left(\mathcal{A}_{\mathbf{x}}\right)\right)$. Let $\bar{s}_{\mathbf{x}}^{q}: H^{q}\left(\mathrm{E}_{\mathbf{y}}^{n \cdot \ell} ; \mathcal{L}_{\mathbf{y}}\right) \rightarrow H^{q}\left(\mathrm{M}_{\mathbf{x}} ; \mathcal{L}_{\mathbf{x}}\right)$ denote the map in cohomology induced by $s_{\mathbf{x}}^{\bullet}: K^{\bullet}\left(\mathcal{B}_{\mathbf{y}}^{n, \ell}\right) \rightarrow K^{\bullet}\left(\mathcal{A}_{\mathbf{x}}\right)$. If $\Gamma \in \pi_{1}(\mathrm{~B}(\mathcal{G}), \mathbf{y})$ is compatible with $\gamma \in \pi_{1}(\mathrm{~B}(\mathcal{T}), \mathbf{x})$, then Theorem 4.8 implies that, up to conjugacy, we have $\bar{s}_{\mathbf{x}}^{q} \circ \Psi_{\mathcal{G}}^{q}(\Gamma)=\Psi_{\mathcal{T}}^{q}(\gamma) \circ \bar{s}_{\mathbf{x}}^{q}$ for each $q, 0 \leq q \leq \ell$. We will pursue the implications of this relationship for Gauss-Manin connections in Section 7 .

\section{Compatible Classes}

For the type $\mathcal{G}$ of general position arrangements, the closure of the moduli space is $\overline{\mathrm{B}}(\mathcal{G})=\left(\mathbb{C P}^{\ell}\right)^{n}$. Recall that points in $\left(\mathbb{C P}^{\ell}\right)^{n}$ are given by matrices $\mathbf{x}$ of the form (3.1), and that $\Delta_{J}(\mathbf{x})$ denotes the determinant of the submatrix of $\mathbf{x}$ with rows specified by $\ell+1$ element subsets $J=\left\{j_{1}, \ldots, j_{\ell+1}\right\}$ of $[n+1]=\{1, \ldots, n+1\}$. The divisor $\mathrm{D}(\mathcal{G})=\overline{\mathrm{B}}(\mathcal{G}) \backslash \mathrm{B}(\mathcal{G})$ is given by $\mathrm{D}(\mathcal{G})=\bigcup_{J} \mathrm{D}_{J}$, whose components, $\mathrm{D}_{J}=\left\{\mathbf{x} \in\left(\mathbb{C P}^{\ell}\right)^{n} \mid \Delta_{J}(\mathbf{x})=0\right\}$, are irreducible hypersurfaces indexed by $J$.

Choose a basepoint $\mathbf{y} \in \mathrm{B}(\mathcal{G})$, and for each $\ell+1$ element subset $J$ of $[n+1]$, let $\mathbf{z}_{J}$ be a generic point in $\mathrm{D}_{J}$. Let $\Gamma_{J}$ be a meridian loop based at $\mathbf{y}$ in $\mathrm{B}(\mathcal{G})$ about the point $\mathbf{z}_{J} \in \mathrm{D}_{J}$. Note that $\mathbf{y} \in \mathrm{Y}(\mathcal{T})$ and that $\Gamma_{J}$ is a (possibly null-homotopic) loop in $\mathrm{Y}(\mathcal{T})$ for any combinatorial type $\mathcal{T}$.

Proposition 5.1. For any combinatorial type $\mathcal{T}$, the homology group $H_{1}(\mathrm{Y}(\mathcal{T}))$ is generated by the classes $\left\{\left[\Gamma_{J}\right] \mid J \in \operatorname{ind}(\mathcal{T})\right\}$.

Proof. Given $\mathcal{T}$, let $Z=\bigcup_{J \in \operatorname{ind}(\mathcal{T})} \mathrm{D}_{J}$, so that $\mathrm{Y}(\mathcal{T})=\left(\mathbb{C P}^{\ell}\right)^{n} \backslash Z$. Denote by $\Sigma Z$ the singular set of $Z$, and let $X=\left(\mathbb{C P}^{\ell}\right)^{n} \backslash \Sigma Z$ and $D=Z \backslash \Sigma Z$. Consider the corresponding Gysin sequence in homology with integer coefficients, as discussed in [9. p. 46]. This sequence is of the form

$$
\cdots \rightarrow H_{k-1}(D) \stackrel{\theta}{\rightarrow} H_{k}(X \backslash D) \stackrel{i_{*}}{\rightarrow} H_{k}(X) \rightarrow H_{k-2}(D) \stackrel{\theta}{\rightarrow} H_{k-1}(X \backslash D) \rightarrow \ldots
$$

where $i: X \backslash D \rightarrow X$ denotes the inclusion.

Since the complex codimension of $\Sigma Z$ is at least two in $\left(\mathbb{C P}^{\ell}\right)^{n}$, the inclusion of $X$ in $\left(\mathbb{C P}^{\ell}\right)^{n}$ induces an isomorphism $H_{1}(X) \simeq H_{1}\left(\left(\mathbb{C P}^{\ell}\right)^{n}\right.$ and thus $H_{1}(X)=0$. So for small $k$, the above sequence is of the form

$$
\cdots \rightarrow H_{2}(X) \rightarrow H_{0}(D) \stackrel{\theta}{\rightarrow} H_{1}(X \backslash D) \rightarrow H_{1}(X)=0
$$

The connected components of $D=Z \backslash \Sigma Z$ are in one-to-one correspondence with the irreducible components $\mathrm{D}_{J}$ of $Z$. Hence, $H_{0}(D)$ is free abelian, of rank equal to the cardinality of ind $(\mathcal{T})$. A basis for $H_{0}(D)$ is given by the classes $\left[\mathbf{z}_{J}\right], J \in \operatorname{ind}(\mathcal{T})$, where $\mathbf{z}_{J} \in \mathrm{D}_{J}$ is the generic point in $\mathrm{D}_{J}$ chosen above. Thus the classes $\theta\left(\left[\mathbf{z}_{J}\right]\right)$ generate $H_{1}(X \backslash D)=H_{1}(\mathrm{Y}(\mathcal{T}))$.

Finally, let $T$ be a tubular neighborhood of $D$, and let $p: \partial T \rightarrow D$ denote the projection from the boundary of $T$ to $D$. Then the map $\theta: H_{0}(D) \rightarrow H_{1}(X \backslash D)$ 
is given by $\theta([\mathbf{z}])=\left[p^{-1}(\mathbf{z})\right]$. Since $\left[p^{-1}\left(\mathbf{z}_{J}\right)\right]$ clearly coincides with $\left[\Gamma_{J}\right]$ up to sign for each $J$, this completes the proof.

Corollary 5.2. The homology group $H_{1}(\mathrm{~B}(\mathcal{G}))$ is generated by the classes $\left[\Gamma_{J}\right]$, where $J$ ranges over all $\ell+1$ element subsets of $[n+1]$.

For a combinatorial type $\mathcal{T}$, recall that $\operatorname{dep}(\mathcal{T})$ denotes the set of all $\ell+1$ element subsets $J=\left\{j_{1}, \ldots, j_{\ell+1}\right\}$ of $[n+1]$ for which the determinant $\Delta_{J}(\mathbf{x})$ vanishes for $\mathbf{x} \in \mathrm{B}(\mathcal{T})$. If $\mathcal{T}$ is realizable, $\operatorname{dep}(\mathcal{T})$ is the set of all $J$ for which $\left\{H_{j_{1}}, \ldots, H_{j_{\ell+1}}\right\}$ is linearly dependent in the projective closure of an arrangement $\mathcal{A}$ of type $\mathcal{T}$. Impose a partial order on combinatorial types as follows: $\mathcal{T} \geq \mathcal{T}^{\prime} \Longleftrightarrow \operatorname{dep}(\mathcal{T}) \subseteq \operatorname{dep}\left(\mathcal{T}^{\prime}\right)$. Note that the combinatorial type $\mathcal{G}$ of general position arrangements is the maximal element with respect to this partial order.

Write $\mathcal{T}>\mathcal{T}^{\prime}$ if $\operatorname{dep}(\mathcal{T}) \subsetneq \operatorname{dep}\left(\mathcal{T}^{\prime}\right)$. In this case we define the relative dependence set $\operatorname{dep}\left(\mathcal{T}^{\prime}, \mathcal{T}\right)=\operatorname{dep}\left(\mathcal{T}^{\prime}\right) \backslash \operatorname{dep}(\mathcal{T})$. If $\mathcal{T}>\mathcal{T}^{\prime}$, we say that $\mathcal{T}$ covers $\mathcal{T}^{\prime}$ and $\mathcal{T}^{\prime}$ is a degeneration of $\mathcal{T}$ if there is no combinatorial type $\mathcal{T}^{\prime \prime}$ with $\mathcal{T}>\mathcal{T}^{\prime \prime}>\mathcal{T}^{\prime}$.

Lemma 5.3. The moduli space $\mathrm{B}\left(\mathcal{T}^{\prime}\right)$ has complex codimension one in the closure $\overline{\mathrm{B}}(\mathcal{T})$ of the moduli space $\mathrm{B}(\mathcal{T})$ if and only if $\mathcal{T}$ covers $\mathcal{T}^{\prime}$.

Proof. If $\mathrm{B}\left(\mathcal{T}^{\prime}\right)$ is codimension one in $\overline{\mathrm{B}}(\mathcal{T})$, then clearly $\mathcal{T}$ covers $\mathcal{T}^{\prime}$. Conversely, if $\mathcal{T}$ covers $\mathcal{T}^{\prime}$, then $\mathrm{B}\left(\mathcal{T}^{\prime}\right) \subset \overline{\mathrm{B}}(\mathcal{T})$ is defined by the vanishing of $\Delta_{J}$ for any $J \in \operatorname{dep}\left(\mathcal{T}^{\prime}, \mathcal{T}\right)$.

Recall that $j_{\mathcal{T}}: \mathrm{B}(\mathcal{T}) \rightarrow \mathrm{Y}(\mathcal{T})$ denotes the natural inclusion.

Theorem 5.4. Let $\mathcal{T}$ be a combinatorial type which covers the type $\mathcal{T}^{\prime}$. Let $\mathbf{x}^{\prime}$ be a point in $\mathrm{B}\left(\mathcal{T}^{\prime}\right)$, and $\gamma \in \pi_{1}(\mathrm{~B}(\mathcal{T}), \mathbf{x})$ a simple loop in $\mathrm{B}(\mathcal{T})$ about $\mathbf{x}^{\prime}$. Then the homology class $[\gamma]$ satisfies

$$
\left(j_{\mathcal{T}}\right)_{*}([\gamma])=\sum_{J \in \operatorname{dep}\left(\mathcal{T}^{\prime}, \mathcal{T}\right)} m_{J} \cdot\left[\Gamma_{J}\right]
$$

where $m_{J}$ is the order of vanishing of the restriction of $\Delta_{J}$ to $\overline{\mathrm{B}}(\mathcal{T})$ along $\mathrm{B}\left(\mathcal{T}^{\prime}\right)$.

Proof. By Proposition 5.1, the classes $\left\{\left[\Gamma_{J}\right] \mid J \in \operatorname{ind}(\mathcal{T})\right\}$ generate $H_{1}(\mathrm{Y}(\mathcal{T}))$. So the image of $[\gamma] \in H_{1}(\mathrm{~B}(\mathcal{T}))$ in $H_{1}(\mathrm{Y}(\mathcal{T}))$ is of the form $\left(j_{\mathcal{T}}\right)_{*}([\gamma])=\sum m_{J} \cdot\left[\Gamma_{J}\right]$, where the sum is over all $J \in \operatorname{ind}(\mathcal{T})$, and $m_{J} \in \mathbb{Z}$.

To determine the coefficients $m_{J}$, let $f=0$ be a local defining equation for $\mathrm{B}\left(\mathcal{T}^{\prime}\right)$ in $\overline{\mathrm{B}}(\mathcal{T})$ near $\mathbf{x}$. By hypothesis, the winding number of $f$ about $\gamma$ is one:

$$
\frac{1}{2 \pi \mathrm{i}} \int_{\gamma} \frac{d f}{f}=1 .
$$

For each $J \in \operatorname{ind}(\mathcal{T})$, let $B_{J}$ be a disk with $\partial B_{J}=\gamma, B_{J} \cap \mathrm{B}\left(\mathcal{T}^{\prime}\right)=\mathbf{x}^{\prime}$, and $B_{J} \backslash\left\{\mathbf{x}^{\prime}\right\} \subset \mathrm{B}(\mathcal{T})$. Suppose also that $F_{J}:\left(\mathbb{C P}^{\ell}\right)^{n} \rightarrow \mathbb{C}$ defines the divisor $\mathrm{D}_{J}=$ $\left\{\Delta_{J}=0\right\}$ locally near $\mathbf{x}$, and let $\bar{j}_{\mathcal{T}}: \overline{\mathrm{B}}(\mathcal{T}) \rightarrow\left(\mathbb{C P}^{\ell}\right)^{n}$ denote the inclusion of the closure of $\mathrm{B}(\mathcal{T})$ in $\left(\mathbb{C P}^{\ell}\right)^{n}$. The coefficient $m_{J}$ is then given by the winding number of $F_{J} \circ \bar{j}_{\mathcal{T}}$ about $\gamma$ :

$$
m_{J}=\frac{1}{2 \pi \mathrm{i}} \int_{\gamma} \frac{d\left(F_{J} \circ \bar{j}_{\mathcal{T}}\right)}{F_{J} \circ \bar{j}_{\mathcal{T}}}
$$


If $J \in \operatorname{ind}\left(\mathcal{T}^{\prime}\right) \cap \operatorname{ind}(\mathcal{T})$, then $F_{J}\left(\mathbf{x}^{\prime}\right) \neq 0$. So for such $J$, the restriction of $F_{J}$ to the disk $B_{J}$ is never zero. Consequently, we have $\int_{\gamma} d\left(F_{J} \circ \bar{j}_{\mathcal{T}}\right) / F_{J} \circ \bar{j}_{\mathcal{T}}=0$, and thus $m_{J}=0$ if $J \in \operatorname{ind}\left(\mathcal{T}^{\prime}\right)$.

If $J \in \operatorname{dep}\left(\mathcal{T}^{\prime}, \mathcal{T}\right)$, then the restriction, $F_{J} \circ \bar{j}_{\mathcal{T}}$, of $F_{J}$ to $\overline{\mathrm{B}}(\mathcal{T})$ defines a hypersurface which contains $\mathrm{B}\left(\mathcal{T}^{\prime}\right)$. So near $\mathbf{x}$, we have $F_{J} \circ \bar{j}_{\mathcal{T}}=c f^{m}$, where $c \in \mathbb{C}^{*}$, and $m \in \mathbb{Z}_{>0}$ is the order of vanishing of $F_{J} \circ \bar{j}_{\mathcal{T}}$ along $\mathrm{B}\left(\mathcal{T}^{\prime}\right)$. Hence for $J \in \operatorname{dep}\left(\mathcal{T}^{\prime}, \mathcal{T}\right)$, the coefficient

$$
m_{J}=\frac{1}{2 \pi \mathrm{i}} \int_{\gamma} \frac{d\left(F_{J} \circ \bar{j}_{\mathcal{T}}\right)}{F_{J} \circ \bar{j}_{\mathcal{T}}}=\frac{1}{2 \pi \mathrm{i}} \int_{\gamma} \frac{d\left(c f^{m}\right)}{c f^{m}}=\frac{m}{2 \pi \mathrm{i}} \int_{\gamma} \frac{d f}{f}=m
$$

is as asserted.

Recall that we say $\Gamma \in \pi_{1}(\mathrm{~B}(\mathcal{G}), \mathbf{y})$ is compatible with $\gamma \in \pi_{1}(\mathrm{~B}(\mathcal{T}), \mathbf{x})$ if the homology classes $[\Gamma]$ and $[\gamma]$ satisfy $\left(i_{\mathcal{T}}\right)_{*}([\Gamma])=\left(j_{\mathcal{T}}\right)_{*}([\gamma])$.

Corollary 5.5. If $\mathcal{T}$ covers $\mathcal{T}^{\prime}$ and $\gamma \in \pi_{1}(\mathrm{~B}(\mathcal{T}), \mathbf{x})$ is a simple loop in $\mathrm{B}(\mathcal{T})$ about $\mathbf{x}^{\prime} \in \mathrm{B}\left(\mathcal{T}^{\prime}\right)$, then any loop $\Gamma \in \pi_{1}(\mathrm{~B}(\mathcal{G}), \mathbf{y})$ whose homology class satisfies the condition $[\Gamma]=\sum_{J} m_{J} \cdot\left[\Gamma_{J}\right]$ given by (5.1) is compatible with $\gamma$.

\section{Nonresonant Weights}

If $\mathcal{L}$ is a nontrivial rank one local system on the complement $\mathrm{E}^{n, \ell}$ of a general position arrangement $\mathcal{B}^{n, \ell}$ in $\mathbb{C}^{\ell}$, then the cohomology $H^{q}\left(\mathrm{E}^{n, \ell} ; \mathcal{L}\right)$ vanishes in all dimensions $q$ except possibly $q=\ell$. See 3 , §7] for a proof of this fact using the complex $K^{\bullet}\left(\mathcal{B}^{n, \ell}\right)$. For an arbitrary arrangement $\mathcal{A}$, a collection of weights $\lambda$ and the corresponding local system $\mathcal{L}$ are called nonresonant if the cohomology $H^{q}(\mathrm{M} ; \mathcal{L})$ vanishes in all dimensions $q$ except possibly $q=\ell$. We now recall a combinatorial condition due to Schechtman, Terao, and Varchenko [20] which insures nonresonance. An edge of an arrangement $\mathcal{A}$ is a nonempty intersection of hyperplanes in $\mathcal{A}$. An edge is called dense if the subarrangement of hyperplanes containing it is irreducible: the hyperplanes cannot be partitioned into nonempty sets so that, after a change of coordinates, hyperplanes in different sets are in different coordinates. This is a combinatorially determined property, see [20]. For each edge $X$, define $\lambda_{X}=\sum_{X \subseteq H_{j}} \lambda_{j}$. Let $\mathcal{A}_{\infty}=\mathcal{A} \cup H_{n+1}$ be the projective closure of $\mathcal{A}$, the union of $\mathcal{A}$ and the hyperplane at infinity in $\mathbb{C P}^{\ell}$, see [18]. Set $\lambda_{n+1}=-\sum_{j=1}^{n} \lambda_{j}$.

Theorem 6.1 (20]). Let $\mathrm{M}$ be the complement of an essential arrangement $\mathcal{A}$ in $\mathbb{C}^{\ell}$ of combinatorial type $\mathcal{T}$. If $\mathcal{L}$ is a rank one local system on $\mathrm{M}$ whose weights $\lambda$ satisfy the condition

$$
\lambda_{X} \notin \mathbb{Z}_{\geq 0} \text { for every dense edge } X \text { of } \mathcal{A}_{\infty},
$$

then $H^{q}(\mathrm{M} ; \mathcal{L})=0$ for $q \neq \ell$ and $\operatorname{dim} H^{\ell}(\mathrm{M} ; \mathcal{L})=|\chi(\mathrm{M})|$, where $\chi(\mathrm{M})$ is the Euler characteristic of $\mathrm{M}$.

Thus the (STV)-condition implies nonresonance. In this paper, we call local systems $\mathcal{L}$ whose weights $\lambda$ satisfy the (STV)-condition $\mathcal{T}$-nonresonant.

Remark 6.2. The hyperplanes are among the dense edges for any arrangement, and are the only dense edges for a general position arrangement. Consequently, if $\lambda$ is $\mathcal{T}$-nonresonant, then $\lambda$ is $\mathcal{G}$-nonresonant. 
Any collection of weights $\lambda$ determines an element $a_{\lambda}=\sum_{j=1}^{n} \lambda_{j} a_{j} \in A^{1}(\mathcal{A})$ in the Orlik-Solomon algebra of $\mathcal{A}$. Since $a_{\lambda} \wedge a_{\lambda}=0$, multiplication by $a_{\lambda}$ defines a differential on $A(\mathcal{A})$. The resulting complex $\left(A^{\bullet}(\mathcal{A}), a_{\lambda} \wedge\right)$ may be identified with a subcomplex of the twisted de Rham complex of $\mathrm{M}$ with coefficients in $\mathcal{L}$. Theorem 6.1 above may be established by showing that, for $\mathcal{T}$-nonresonant weights $\lambda$, there is an isomorphism $H^{*}(\mathrm{M} ; \mathcal{L}) \simeq H^{*}\left(A^{\bullet}(\mathcal{A}), a_{\lambda} \wedge\right)$ [10, 20], and that $H^{q}\left(A^{\bullet}(\mathcal{A}), a_{\lambda} \wedge\right)=$ 0 for $q \neq \ell$ and $\operatorname{dim} H^{\ell}\left(A^{\bullet}(\mathcal{A}), a_{\lambda} \wedge\right)=|\chi(\mathrm{M})|$, see [24].

For $\mathcal{T}$-nonresonant weights, one can exhibit an explicit basis, the $\beta \mathbf{n b c}$ basis of 11, for the single nonvanishing cohomology group $H^{\ell}(\mathrm{M} ; \mathcal{L}) \simeq H^{\ell}\left(A^{\bullet}(\mathcal{A}), a_{\lambda} \wedge\right)$. Recall that the hyperplanes of $\mathcal{A}=\left\{H_{j}\right\}_{j=1}^{n}$ are ordered. A circuit is an inclusionminimal dependent set of hyperplanes in $\mathcal{A}$, and a broken circuit is a set $S$ for which there exists $H<\min (S)$ so that $S \cup\{H\}$ is a circuit. A frame is a maximal independent set, and an nbc frame is a frame which contains no broken circuit. Since $\mathcal{A} \subset \mathbb{C}^{\ell}$ is essential, every frame has cardinality $\ell$. An nbc frame $B=$ $\left(H_{j_{1}}, \ldots, H_{j_{\ell}}\right)$ is a $\beta \mathbf{n b c}$ frame provided that for each $k, 1 \leq k \leq \ell$, there exists $H \in \mathcal{A}$ such that $H<H_{j_{k}}$ and $\left(B \backslash\left\{H_{j_{k}}\right\}\right) \cup\{H\}$ is a frame.

Let $\beta \operatorname{nbc}(\mathcal{A})$ be the set of all $\beta \mathbf{n b c}$ frames of $\mathcal{A}$. Given $B=\left(H_{j_{1}}, \ldots, H_{j_{\ell}}\right)$ in $\beta \operatorname{nbc}(\mathcal{A})$, define $\zeta(B) \in A^{\ell}(\mathcal{A})$ by $\zeta(B)=\wedge_{p=1}^{\ell} a_{\lambda}\left(X_{p}\right)$, where $X_{p}=\bigcap_{k=p}^{\ell} H_{j_{k}}$ and $a_{\lambda}(X)=\sum_{X \subseteq H_{i}} \lambda_{i} a_{i}$. Denote the cohomology class of $\zeta(B)$ in $H^{\ell}\left(A^{\bullet}(\mathcal{A}), a_{\lambda} \wedge\right)$ by the same symbol.

Theorem 6.3 (《11). Let $\mathrm{M}$ be the complement of an essential arrangement $\mathcal{A}$ in $\mathbb{C}^{\ell}$ of combinatorial type $\mathcal{T}$. If $\mathcal{L}$ is a rank one local system on $\mathrm{M}$ corresponding to $\mathcal{T}$-nonresonant weights $\lambda$, then the set $\{\zeta(B) \mid B \in \beta \mathbf{n b c}(\mathcal{A})\}$ is a basis for the only nontrivial local system cohomology group $H^{\ell}(\mathrm{M} ; \mathcal{L}) \simeq H^{\ell}\left(A^{\bullet}(\mathcal{A}), a_{\lambda} \wedge\right)$.

Example 6.4. If $\mathcal{B}^{n, \ell}$ is a general position arrangement of $n$ hyperplanes in $\mathbb{C}^{\ell}$, then $\beta \mathbf{n b c}\left(\mathcal{B}^{n, \ell}\right)=\left\{\left(H_{j_{1}}, \ldots, H_{j_{\ell}}\right) \mid 2 \leq j_{1}<\cdots<j_{\ell} \leq n\right\}$. For $\mathcal{G}$-nonresonant weights $\lambda$, it follows that the $\beta$ nbc basis for $H^{\ell}\left(\mathrm{E}^{n, \ell} ; \mathcal{L}\right)$ consists of monomials $\eta_{T}=$ $\lambda_{j_{1}} \cdots \lambda_{j_{\ell}} a_{T}$, where $T=\left\{j_{1}, \ldots, j_{\ell}\right\}, 2 \leq j_{1}<\cdots<j_{\ell} \leq n$, and $a_{T}=a_{j_{1}} \wedge \cdots \wedge a_{j_{\ell}}$.

Theorem 6.5. For $\mathcal{T}$-nonresonant weights $\lambda$, the map $s: \mathrm{M} \rightarrow \mathrm{E}^{n}$ induces a surjection $s^{*}: H^{\ell}\left(\mathrm{E}^{n, \ell}, \mathcal{L}\right) \rightarrow H^{\ell}(\mathrm{M} ; \mathcal{L})$.

Proof. The map $s: \mathrm{M} \rightarrow \mathrm{E}^{n}$ induces a surjection $s^{*}: A\left(\mathcal{B}^{n, \ell}\right) \rightarrow A(\mathcal{A})$ by Proposition 2.2. This is a chain map $s^{*}:\left(A^{\bullet}\left(\mathcal{B}^{n, \ell}\right), a_{\lambda} \wedge\right) \rightarrow\left(A^{\bullet}(\mathcal{A}), a_{\lambda} \wedge\right)$, as is readily checked. So there is an induced map $\bar{s}: H^{\ell}\left(A^{\bullet}\left(\mathcal{B}^{n, \ell}\right), a_{\lambda} \wedge\right) \rightarrow H^{\ell}\left(A^{\bullet}(\mathcal{A}), a_{\lambda} \wedge\right)$ in cohomology.

By Remark 6.2, if $\lambda$ is $\mathcal{T}$-nonresonant, then $\lambda$ is $\mathcal{G}$-nonresonant. For such weights, we have isomorphisms $H^{\ell}\left(\mathrm{E}^{n, \ell} ; \mathcal{L}\right) \simeq H^{\ell}\left(A^{\bullet}\left(\mathcal{B}^{n, \ell}\right), a_{\lambda} \wedge\right)$ and $H^{\ell}(\mathrm{M} ; \mathcal{L}) \simeq$ $H^{\ell}\left(A^{\bullet}(\mathcal{A}), a_{\lambda} \wedge\right)$. Furthermore, the elements of the $\beta \mathbf{n b c}$ basis for $H^{\ell}\left(A^{\bullet}(\mathcal{A}), a_{\lambda} \wedge\right)$ are linear combinations of the monomials $\eta_{T}$, which form the $\beta$ nbc basis for $H^{\ell}\left(A^{\bullet}\left(\mathcal{B}^{n, \ell}\right), a_{\lambda} \wedge\right)$ as noted in Example 6.4. This fact, together with the surjectivity of the map $s^{*}: A\left(\mathcal{B}^{n, \ell}\right) \rightarrow A(\mathcal{A})$ completes the proof.

This leads to the following consequence of Theorem 4.8.

Corollary 6.6. Let $\lambda$ be a collection of $\mathcal{T}$-nonresonant weights. If $\gamma \in \pi_{1}(\mathrm{~B}(\mathcal{T}), \mathbf{x})$ and $\Gamma \in \pi_{1}(\mathrm{~B}(\mathcal{G}), \mathbf{y})$ are compatible, then the conjugacy class of the automorphism $\Psi_{\mathcal{T}}^{\ell}(\gamma) \in \operatorname{Aut}_{\mathbb{C}}\left(H^{\ell}\left(\mathrm{M}_{\mathbf{x}} ; \mathcal{L}_{\mathbf{x}}\right)\right)$ is determined by $\Psi_{\mathcal{G}}^{\ell}(\Gamma) \in \operatorname{Aut}_{\mathbb{C}}\left(H^{\ell}\left(\mathrm{E}_{\mathbf{y}}^{n, \ell} ; \mathcal{L}_{\mathbf{y}}\right)\right)$ and the surjection $\bar{s}_{\mathbf{x}}^{\ell}: H^{\ell}\left(\mathrm{E}_{\mathbf{y}}^{n, \ell} ; \mathcal{L}_{\mathbf{y}}\right) \rightarrow H^{\ell}\left(\mathrm{M}_{\mathbf{x}} ; \mathcal{L}_{\mathbf{x}}\right)$. 


\section{Gauss-Manin Connections}

The vector bundle $\pi^{q}: \mathbf{H}^{q} \rightarrow \mathrm{B}(\mathcal{T})$, with fiber $\left(\pi^{q}\right)^{-1}(\mathbf{x})=H^{q}\left(\mathrm{M}_{\mathbf{x}} ; \mathcal{L}_{\mathbf{x}}\right)$ at $\mathbf{x} \in \mathrm{B}(\mathcal{T})$, supports a Gauss-Manin connection corresponding to the representation $\Psi_{\mathcal{T}}^{q}: \pi_{1}(\mathrm{~B}(\mathcal{T}), \mathbf{x}) \rightarrow \operatorname{Aut}_{\mathbb{C}}\left(H^{q}\left(\mathrm{M}_{\mathbf{x}} ; \mathcal{L}_{\mathbf{x}}\right)\right)$. We now study this connection using results of the previous section. In light of Corollary 6.6, for an arrangement $\mathcal{A}$ of combinatorial type $\mathcal{T}$, we focus on $\mathcal{T}$-nonresonant weights and the case $q=\ell$.

Over a manifold, there is a well known equivalence between local systems and complex vector bundles equipped with flat connections, see [8, 16]. Let $\mathbf{V} \rightarrow X$ be such a bundle, with connection $\nabla$. The latter is a $\mathbb{C}$-linear map $\nabla: \mathcal{E}^{0}(\mathbf{V}) \rightarrow \mathcal{E}^{1}(\mathbf{V})$, where $\mathcal{E}^{p}(\mathbf{V})$ denotes the complex $p$-forms on $X$ with values in $\mathbf{V}$, which satisfies $\nabla(f \sigma)=\sigma d f+f \nabla(\sigma)$ for a function $f$ and $\sigma \in \mathcal{E}^{0}(\mathbf{V})$. The connection extends to a map $\nabla: \mathcal{E}^{p}(\mathbf{V}) \rightarrow \mathcal{E}^{p+1}(\mathbf{V})$ for $p \geq 0$, and is flat if the curvature $\nabla \circ \nabla$ vanishes. Call two connections $\nabla$ and $\nabla^{\prime}$ on $\mathbf{V}$ isomorphic if $\nabla^{\prime}$ is obtained from $\nabla$ by a gauge transformation, $\nabla^{\prime}=g \circ \nabla \circ g^{-1}$ for some $g: X \rightarrow \operatorname{Hom}(\mathbf{V}, \mathbf{V})$.

The aforementioned equivalence is given by $(\mathbf{V}, \nabla) \mapsto \mathbf{V}^{\nabla}$, where $\mathbf{V}^{\nabla}$ is the local system, or locally constant sheaf, of horizontal sections $\left\{\sigma \in \mathcal{E}^{0}(\mathbf{V}) \mid \nabla(\sigma)=0\right\}$. There is also a well known equivalence between local systems on $X$ and finite dimensional complex representations of the fundamental group of $X$. Note that isomorphic connections give rise to the same representation. Under these equivalences, the local system on $X=\mathrm{B}(\mathcal{T})$ induced by the representation $\Psi_{\mathcal{T}}^{q}$ corresponds to a flat connection on the vector bundle $\pi^{q}: \mathbf{H}^{q} \rightarrow \mathrm{B}(\mathcal{T})$, the Gauss-Manin connection.

For $\mathcal{T}$-nonresonant weights, the $\beta$ nbc basis of Theorem 6.3 provides a basis for each fiber $H^{\ell}\left(\mathrm{M}_{\mathbf{x}} ; \mathcal{L}_{\mathbf{x}}\right)$, independent of $\mathbf{x}$. Thus the vector bundle $\pi^{\ell}: \mathbf{H}^{\ell} \rightarrow$ $\mathrm{B}(\mathcal{T})$ is trivial, see [11, 22]. Let $\gamma \in \pi_{1}(\mathrm{~B}(\mathcal{T}), \mathbf{x})$, and let $g: \mathbb{S}^{1} \rightarrow \mathrm{B}(\mathcal{T})$ be a representative loop. Pulling back the trivial bundle $\pi^{\ell}: \mathbf{H}^{\ell} \rightarrow \mathrm{B}(\mathcal{T})$ and the Gauss-Manin connection $\nabla$, we obtain a flat connection $g^{*}(\nabla)$ on the trivial vector bundle over the circle corresponding to the representation of $\pi_{1}\left(\mathbb{S}^{1}, 1\right)=\langle\zeta\rangle=\mathbb{Z}$ given by $\zeta \mapsto \Psi_{\mathcal{T}}^{\ell}(\gamma)$. Specifying the flat connection $g^{*}(\nabla)$ amounts to choosing a logarithm of $\Psi_{\mathcal{T}}^{\ell}(\gamma)$. The connection $g^{*}(\nabla)$ is determined by a connection 1 -form $d z / z \otimes \Omega_{\mathcal{T}}(\gamma)$, where the connection matrix $\Omega_{\mathcal{T}}(\gamma)$ corresponding to $\gamma$ satisfies $\Psi_{\mathcal{T}}^{\ell}(\gamma)=\exp \left(-2 \pi \mathrm{i} \Omega_{\mathcal{T}}(\gamma)\right)$. If $\gamma$ and $\hat{\gamma}$ are conjugate in $\pi_{1}(\mathrm{~B}(\mathcal{T}), \mathbf{x})$, then the resulting connection matrices are conjugate, and the corresponding connections on the trivial vector bundle over the circle are isomorphic. In this sense, the connection matrix $\Omega_{\mathcal{T}}(\gamma)$ is determined by the homology class $[\gamma]$ of $\gamma$.

Theorem 7.1. Let $\lambda$ be a collection of $\mathcal{T}$-nonresonant weights. If $\gamma \in \pi_{1}(\mathrm{~B}(\mathcal{T}), \mathbf{x})$ and $\Gamma \in \pi_{1}(\mathrm{~B}(\mathcal{G}), \mathbf{y})$ are compatible, then there is a commutative diagram

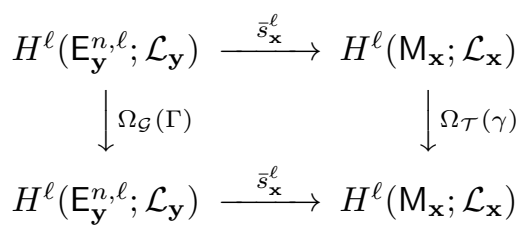

Thus a Gauss-Manin connection matrix $\Omega_{\mathcal{T}}(\gamma)$ is determined by a Gauss-Manin connection matrix $\Omega_{\mathcal{G}}(\Gamma)$ and the surjection $\bar{s}_{\mathbf{x}}^{\ell}: H^{\ell}\left(\mathrm{E}_{\mathbf{y}}^{n, \ell} ; \mathcal{L}_{\mathbf{y}}\right) \rightarrow H^{\ell}\left(\mathrm{M}_{\mathbf{x}} ; \mathcal{L}_{\mathbf{x}}\right)$.

Proof. Choose a connection matrix $\Omega_{\mathcal{G}}(\Gamma)$ so that $\Psi_{\mathcal{G}}^{\ell}(\Gamma)=\exp \left(-2 \pi\right.$ i $\left.\Omega_{\mathcal{G}}(\Gamma)\right)$. Since $\bar{s}_{\mathbf{x}}^{\ell}: H^{\ell}\left(\mathrm{E}_{\mathbf{y}}^{n, \ell} ; \mathcal{L}_{\mathbf{y}}\right) \rightarrow H^{\ell}\left(\mathrm{M}_{\mathbf{x}} ; \mathcal{L}_{\mathbf{x}}\right)$ is surjective by Theorem 6.5, the endomorphism $\Omega_{\mathcal{G}}(\Gamma): H^{\ell}\left(\mathrm{E}_{\mathbf{y}}^{n, \ell} ; \mathcal{L}_{\mathbf{y}}\right) \rightarrow H^{\ell}\left(\mathrm{E}_{\mathbf{y}}^{n, \ell} ; \mathcal{L}_{\mathbf{y}}\right)$ induces an endomorphism $H^{\ell}\left(\mathrm{M}_{\mathbf{x}} ; \mathcal{L}_{\mathbf{x}}\right) \rightarrow$ 
$H^{\ell}\left(\mathrm{M}_{\mathbf{x}} ; \mathcal{L}_{\mathbf{x}}\right)$, which we denote by $\Omega_{\mathcal{T}}(\gamma)$. We assert that $\Omega_{\mathcal{T}}(\gamma)$ is a connection matrix for $\Psi_{\mathcal{T}}^{\ell}(\gamma)$. For this, by the above discussion, it suffices to show that $\exp \left(-2 \pi \mathrm{i} \Omega_{\mathcal{T}}(\gamma)\right)$ is conjugate to $\Psi_{\mathcal{T}}^{\ell}(\gamma)$.

By construction, we have $\bar{s}_{\mathbf{x}}^{\ell} \circ \Omega_{\mathcal{G}}(\Gamma)=\Omega_{\mathcal{T}}(\gamma) \circ \bar{s}_{\mathbf{x}}^{\ell}$. From this it follows that $\bar{s}_{\mathbf{x}}^{\ell} \circ \exp \left(-2 \pi \mathrm{i} \Omega_{\mathcal{G}}(\Gamma)\right)=\exp \left(-2 \pi \mathrm{i} \Omega_{\mathcal{T}}(\gamma)\right) \circ \bar{s}_{\mathbf{x}}^{\ell}$. By Theorem 4.8 and Corollary 6.6 , up to conjugacy, we have $\bar{s}_{\mathbf{x}}^{\ell} \circ \Psi_{\mathcal{G}}^{\ell}(\Gamma)=\Psi_{\mathcal{T}}^{\ell}(\gamma) \circ \bar{s}_{\mathbf{x}}^{\ell}$. Hence, $\exp \left(-2 \pi \mathrm{i} \Omega_{\mathcal{T}}(\gamma)\right)$ is conjugate to $\Psi_{\mathcal{T}}^{\ell}(\gamma)$.

Next we need formulas of Aomoto and Kita [2], see also [18], for the GaussManin connection matrices in the case of general position arrangements. Let $\mathbf{y} \in$ $\mathrm{B}(\mathcal{G})$, and let $\mathcal{B}_{\mathbf{y}}^{n, \ell}$ be the corresponding arrangement of type $\mathcal{G}$, a general position arrangement of $n$ hyperplanes in $\mathbb{C}^{\ell}$, with complement $\mathrm{E}_{\mathbf{y}}^{n, \ell}$. A system of weights $\lambda=\left(\lambda_{1}, \ldots, \lambda_{n}\right)$ is $\mathcal{G}$-nonresonant if $\lambda_{j} \notin \mathbb{Z}_{\geq 0}$ for each $j, 1 \leq j \leq n+1$. Recall that $\lambda_{n+1}=-\sum_{j=1}^{n} \lambda_{j}$. For such weights, the $\beta \mathbf{n b c}$ basis for the cohomology group $H^{\ell}\left(\mathrm{E}_{\mathbf{y}}^{n, \ell} ; \mathcal{L}_{\mathbf{y}}\right)$ with coefficients in the corresponding local system consists of the monomials $\eta_{I}=\lambda_{i_{1}} \cdots \lambda_{i_{\ell}} a_{I}$, where $I=\left\{i_{1}, \ldots, i_{\ell}\right\}, 2 \leq i_{1}<\cdots<i_{\ell} \leq n$, and $a_{I}=a_{i_{1}} \wedge \cdots \wedge a_{i_{\ell}}$, see Example 6.4.

To state the results of Aomoto and Kita on the Gauss-Manin connection for general position arrangements, we require some notation. We use the formulation of [18, $\S 10.3]$. For $J=\left\{j_{1}, \ldots, j_{m}\right\}$, write $\lambda_{J}=\sum_{j \in J} \lambda_{j}$, and let $J_{p}=\left\{j_{1}, \ldots \widehat{j_{p}}, \ldots, j_{m}\right\}$ for each $p, 1 \leq p \leq m$. If $I, I^{\prime} \subset[n],|I|=\left|I^{\prime}\right|=\ell$, and $\left|I \cap I^{\prime}\right|=\ell-1$, let $\epsilon\left(I, I^{\prime}\right)=(-1)^{p+q}$, where $K=I \cup I^{\prime}, I=K_{p}$, and $I^{\prime}=K_{q}$.

Theorem 7.2 ([2]). For $\mathcal{G}$-nonresonant weights $\lambda$, the Gauss-Manin connection on the (trivial) vector bundle $\pi^{\ell}: \mathbf{H}^{\ell} \rightarrow \mathrm{B}(\mathcal{G})$ has connection 1-form

$$
\sum_{J \subset[n+1]} d \log \mathrm{D}_{J} \otimes \Omega_{\mathcal{G}}\left(\Gamma_{J}\right)
$$

where the sum is over all subsets $J$ of $[n+1]$ of cardinality $\ell+1$ and $d \log \mathrm{D}_{J}$ denotes a 1-form on $\left(\mathbb{C P}^{\ell}\right)^{n}$ with a simple logarithmic pole along the divisor $\mathrm{D}_{J}$. The connection matrices $\Omega_{\mathcal{G}}\left(\Gamma_{J}\right)$, acting on the $\beta$ nbc basis $\left\{\eta_{I} \mid 2 \leq i_{1}<\cdots<i_{\ell} \leq n\right\}$ of $H^{\ell}\left(\mathrm{E}_{\mathbf{y}}^{n, \ell} ; \mathcal{L}_{\mathbf{y}}\right)$, are given by the following formulas.

1. If $J \cap\{1, n+1\}=\emptyset$, then

$$
\Omega_{\mathcal{G}}\left(\Gamma_{J}\right)\left(\eta_{I}\right)= \begin{cases}\sum_{p=1}^{\ell+1} \epsilon\left(I, J_{p}\right) \lambda_{J \backslash J_{p}} \eta_{J_{p}} & \text { if } I \subset J, \\ 0 & \text { otherwise. }\end{cases}
$$

2. If $J=J^{\prime} \cup\{n+1\}$, where $J^{\prime}=\left\{j_{1}, \ldots, j_{\ell}\right\}, 2 \leq j_{1}<\cdots<j_{\ell} \leq n$, then

$$
\Omega_{\mathcal{G}}\left(\Gamma_{J}\right)\left(\eta_{I}\right)= \begin{cases}-\left(\sum_{j \in[n] \backslash I} \lambda_{j}\right) \eta_{I} & \text { if } I=J^{\prime}, \\ -\epsilon\left(I, J^{\prime}\right) \lambda_{I \backslash I \cap J^{\prime}} \eta_{J^{\prime}} & \text { if }\left|I \cap J^{\prime}\right|=\ell-1, \\ 0 & \text { otherwise. }\end{cases}
$$

3. If $J \cap\{1, n+1\}=\{1\}$, then

$$
\Omega_{\mathcal{G}}\left(\Gamma_{J}\right)\left(\eta_{I}\right)= \begin{cases}\left(\sum_{j \in J} \lambda_{j}\right) \eta_{I}-\sum_{\left|I \cap I^{\prime}\right|=\ell-1} \epsilon\left(I, I^{\prime}\right) \lambda_{I \backslash I \cap I^{\prime}} \eta_{I^{\prime}} & \text { if } I=J_{1}, \\ 0 & \text { otherwise. }\end{cases}
$$


4. If $J=J^{\prime \prime} \cup\{1, n+1\}$, where $J^{\prime \prime}=\left\{j_{2}, \ldots, j_{\ell}\right\}, 2 \leq j_{2}<\cdots<j_{\ell} \leq n$, then

$\Omega_{\mathcal{G}}\left(\Gamma_{J}\right)\left(\eta_{I}\right)= \begin{cases}-\lambda_{I \backslash I \cap J} \eta_{I}+\lambda_{I \backslash I \cap J} \sum_{I \cap I^{\prime}=I \cap J} \epsilon\left(I, I^{\prime}\right) \eta_{I^{\prime}} & \text { if } J^{\prime \prime} \subset I, \\ 0 & \text { otherwise. }\end{cases}$

We combine these formulas with Theorem 5.4. Theorem 6.5, and Theorem 7.1 to obtain the main result of this paper. It is a combinatorial algorithm for calculating Gauss-Manin connection matrices for an arbitrary combinatorial type $\mathcal{T}$, and $\mathcal{T}$-nonresonant weights, in terms of those for the type $\mathcal{G}$ of general position arrangements. Let $\mathcal{T}$ be a combinatorial type which covers the type $\mathcal{T}^{\prime}$. Let $\mathbf{x}^{\prime}$ be a point in $\mathrm{B}\left(\mathcal{T}^{\prime}\right)$, and $\gamma \in \pi_{1}(\mathrm{~B}(\mathcal{T}), \mathbf{x})$ a simple loop in $\mathrm{B}(\mathcal{T})$ about $\mathbf{x}^{\prime}$. We agree to write $\Omega_{\mathcal{T}}\left(\mathcal{T}^{\prime}\right)$ in place of $\Omega_{\mathcal{T}}(\gamma)$ in this situation. Similarly, we write $\Omega_{\mathcal{G}}(J)$ in place of $\Omega_{\mathcal{G}}\left(\Gamma_{J}\right)$. Fix $\mathcal{T}$-nonresonant (and hence $\mathcal{G}$-nonresonant) weights $\lambda$. For $\mathbf{x} \in \mathrm{B}(\mathcal{T})$ and $\mathbf{y} \in \mathrm{B}(\mathcal{G})$, by Theorems 6.1 and 6.3 , the corresponding local system cohomology groups of the fibers, $H^{q}\left(\mathrm{M}_{\mathbf{x}} ; \mathcal{L}_{\mathbf{x}}\right)$ and $H^{q}\left(\mathrm{E}_{\mathbf{y}}^{n, \ell} ; \mathcal{L}_{\mathbf{y}}\right)$, depend only on the combinatorial types $\mathcal{T}$ and $\mathcal{G}$ and vanish for $q \neq \ell$. So we write $H^{\ell}\left(\mathrm{M}_{\mathbf{x}} ; \mathcal{L}_{\mathbf{x}}\right)=H^{\ell}(\mathcal{T})$ and $H^{\ell}\left(\mathrm{E}_{\mathbf{y}}^{n, \ell} ; \mathcal{L}_{\mathbf{y}}\right)=H^{\ell}(\mathcal{G})$. Denote the $\beta \mathbf{n b c}$ bases of these local system cohomology groups by $\beta \mathbf{n b c}(\mathcal{T})$ and $\beta \mathbf{n b c}(\mathcal{G})$ respectively. This notation makes it clear that a Gauss-Manin connection matrix depends only on the types involved and it is obtained by a combinatorial formula.

Theorem 7.3. Let $\mathcal{T}$ be a combinatorial type which covers the type $\mathcal{T}^{\prime}$. Let $\lambda$ be a collection of $\mathcal{T}$-nonresonant weights, and let $P(\mathcal{T})$ be the matrix of the surjection $H^{\ell}(\mathcal{G}) \rightarrow H^{\ell}(\mathcal{T})$ in the respective $\beta$ nbc bases. Then a Gauss-Manin connection matrix $\Omega_{\mathcal{T}}\left(\mathcal{T}^{\prime}\right)$ is determined by the matrix equation

$$
P(\mathcal{T}) \cdot \Omega_{\mathcal{T}}\left(\mathcal{T}^{\prime}\right)=\left(\sum_{J \in \operatorname{dep}\left(\mathcal{T}^{\prime}, \mathcal{T}\right)} m_{J} \cdot \Omega_{\mathcal{G}}(J)\right) \cdot P(\mathcal{T})
$$

Illustrations of the algorithm provided by this result are presented in Section 8 .

Remark 7.4. For an arbitrary combinatorial type $\mathcal{T}$, and $\mathcal{T}$-nonresonant weights $\lambda$, Terao [22] shows that the Gauss-Manin connection on the bundle $\pi^{\ell}: \mathbf{H}^{\ell} \rightarrow \mathrm{B}(\mathcal{T})$ is determined by a connection 1-form $\sum d \log \mathrm{D}_{j} \otimes \Omega_{j}$, where $\Omega_{j} \in \operatorname{End}_{\mathbb{C}} H^{\ell}\left(\mathrm{M}_{\mathbf{x}} ; \mathcal{L}_{\mathbf{x}}\right)$, each $d \log \mathrm{D}_{j}$ denotes a 1-form on $\overline{\mathrm{B}}(\mathcal{T})$ with a simple logarithmic pole along the irreducible component $\mathrm{D}_{j}$ of the codimension one divisor $\mathrm{D}(\mathcal{T})=\overline{\mathrm{B}}(\mathcal{T}) \backslash \mathrm{B}(\mathcal{T})$, and the sum is over all such irreducible components. Theorem 7.3 computes the Gauss-Manin connection matrices $\Omega_{j}$ here. Since our work is local, our results are independent of Terao's global theorem.

Remark 7.5. Note that there are two floating inputs in Theorem 7.3: the (STV)condition, which ensures nonresonance, and the $\beta \mathbf{n b c}$ basis, which makes the surjections explicit. Should more relaxed conditions for nonresonance be discovered, and suitable cohomology bases be constructed, where the surjection may be made explicit, our methods will apply equally well in the new setting.

For an arbitrary local system $\mathcal{L}$ of rank greater than one on the complement of an arrangement of type $\mathcal{T}$, the results presented here need not apply, since such a local system is not in general induced by a local system on the complement of a Boolean arrangement. However, if $\mathcal{L}$ is an abelian local system of arbitrary rank, then $\mathcal{L}$ is induced by a local system on the complement of a Boolean arrangement. 
The methods of this paper may be applied in this generality in those instances where $\mathcal{L}$ is nonresonant and a higher rank analogue of Theorem 6.5 holds.

In both of the above situations, analogues of the Aomoto-Kita formulas for $\mathcal{G}^{-}$ nonresonant weights, recorded in Theorem 7.2, would be required to express the Gauss-Manin connection matrices for type $\mathcal{T}$ in terms of those for type $\mathcal{G}$ explicitly.

\section{EXAMPLES}

8.1. Codimension one. The moduli space $B(\mathcal{T})$ of a combinatorial type $\mathcal{T}$ of essential arrangements of $n$ hyperplanes in $\mathbb{C}^{\ell}$ is of codimension one in $\left(\mathbb{C P}^{\ell}\right)^{n}$ if the cardinality of $\operatorname{dep}(\mathcal{T})$ is one, $\operatorname{dep}(\mathcal{T})=\{K\}$, where $K=\left\{k_{1}, \ldots, k_{\ell+1}\right\}$, $1 \leq k_{1}<\cdots<k_{\ell+1} \leq n+1$. For these types, the Gauss-Manin connection was determined by Terao [22]. We now sketch how these results may be recovered using the algorithm of Theorem 7.3.

There are two cases to consider: $n+1 \notin K$ and $n+1 \in K$. By permuting hyperplanes, we may assume that $K=[\ell+1]=\{1, \ldots, \ell+1\}$ if $n+1 \notin K$, and that $K=[n-\ell+1, n+1]=\{n-\ell+1, \ldots, n+1\}$ if $n+1 \in K$. If $n+1 \notin K$, we have $\beta \mathbf{n b c}(\mathcal{T})=\beta \mathbf{n b c}(\mathcal{G}) \backslash\left\{\eta_{F}\right\}$, where $F=[2, \ell+1]=\{2, \ldots, \ell+1\}$, while if $n+1 \in K, \beta \mathbf{n b c}(\mathcal{T})=\beta \mathbf{n b c}(\mathcal{G}) \backslash\left\{\eta_{L}\right\}$, where $L=[n-\ell+1, n]=\{n-\ell+1, \ldots, n\}$. Denote the projection $P(\mathcal{T}): H^{\ell}(\mathcal{G}) \rightarrow H^{\ell}(\mathcal{T})$ of Theorem 7.3 by $P_{F}$ if $n+1 \notin K$ and by $P_{L}$ if $n+1 \in K$. It is readily checked that these projections are given by

$$
\begin{aligned}
& P_{F}\left(\eta_{I}\right)=\eta_{I} \quad \text { if } I \neq F, \quad P_{L}\left(\eta_{I}\right)=\eta_{I} \quad \text { if } I \neq L, \\
& P_{F}\left(\eta_{I}\right)=\sum_{j=2}^{\ell+1} \sum_{q=\ell+2}^{n}(-1)^{j+\ell} \frac{\lambda_{j}}{\lambda_{[\ell+1]}} \eta_{F_{j-1}^{q}} \quad \text { if } I=F, \quad P_{L}\left(\eta_{I}\right)=0 \quad \text { if } I=L,
\end{aligned}
$$

where $F_{j-1}^{q}=F_{j-1} \cup\{q\}=\{2, \ldots, \widehat{j}, \ldots, \ell+1, q\}$ for $\ell+2 \leq q \leq n$.

As shown by Terao [22], the moduli space $B(\mathcal{T})$ is smooth, and the irreducible components of the divisor $\mathrm{D}(\mathcal{T})=\overline{\mathrm{B}}(\mathcal{T}) \backslash \mathrm{B}(\mathcal{T})$ correspond to the combinatorial types $\mathcal{T}^{\prime}$ which are covered by $\mathcal{T}$, and are characterized as follows:

(1) $\operatorname{dep}\left(\mathcal{T}^{\prime}, \mathcal{T}\right)=\{J\}$, where $J \subset[n+1],|J|=\ell+1$, and $|K \cap J| \leq \ell-1$

(2) $\operatorname{dep}\left(\mathcal{T}^{\prime}, \mathcal{T}\right)=\left\{K_{p}^{q} \mid q \in[n+1] \backslash K\right\}$ for each $p, 1 \leq p \leq \ell+1$

(3) $\operatorname{dep}\left(\mathcal{T}^{\prime}, \mathcal{T}\right)=\left\{K_{p}^{q} \mid 1 \leq p \leq \ell+1\right\}$ for each $q \in[n+1] \backslash K$

In types (2) and (3), $K_{p}^{q}=K_{p} \cup\{q\}=\left\{k_{1}, \ldots, \widehat{k}_{p}, \ldots, k_{\ell+1}, q\right\}$ for $1 \leq p \leq \ell+1$ and $q \in[n+1] \backslash K$. If $\ell=1$, type (2) does not appear.

For each $J \in \operatorname{dep}\left(\mathcal{T}^{\prime}, \mathcal{T}\right)$, the order of vanishing of the restriction of $\Delta_{J}$ to $\overline{\mathrm{B}}(\mathcal{T})$ along $\mathrm{B}\left(\mathcal{T}^{\prime}\right)$ is equal to one, $m_{J}=1$, see [18, $\left.\S 10.4\right]$. By Theorem 7.3, a corresponding Gauss-Manin connection matrix $\Omega_{\mathcal{T}}\left(\mathcal{T}^{\prime}\right) \in \operatorname{End}_{\mathbb{C}}\left(H^{\ell}(\mathcal{T})\right)$ satisfies

$$
P(\mathcal{T}) \cdot \Omega_{\mathcal{T}}\left(\mathcal{T}^{\prime}\right)=\left(\sum_{J \in \operatorname{dep}\left(\mathcal{T}^{\prime}, \mathcal{T}\right)} \Omega_{\mathcal{G}}(J)\right) \cdot P(\mathcal{T}),
$$

where $P(\mathcal{T})=P_{F}$ if $n+1 \notin K$ and $P(\mathcal{T})=P_{L}$ if $n+1 \in K$.

It is an exercise in linear algebra to recover Terao's calculation of the connection matrix $\Omega_{\mathcal{T}}\left(\mathcal{T}^{\prime}\right)$ from [22]. We give several small, explicit examples next, and leave this elementary, albeit lengthy, exercise in the general case to the interested reader.

Example 8.2. Let $\mathcal{T}$ be the combinatorial type of the arrangement $\mathcal{A}$ of 4 lines in $\mathbb{C}^{2}$ depicted in Figure 1. Here $\mathrm{B}(\mathcal{T})$ is codimension one in $\left(\mathbb{C P}^{2}\right)^{4}=\overline{\mathrm{B}}(\mathcal{G})$. 

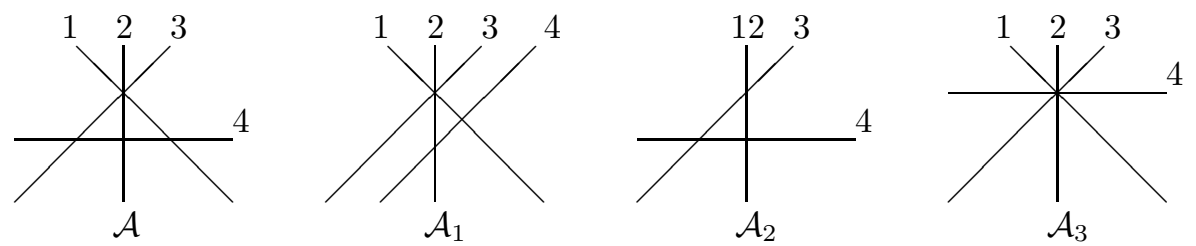

Figure 1. A Codimension One Arrangement and Three Degenerations

A collection of weights $\lambda$ is $\mathcal{T}$-nonresonant if $\lambda_{j}(1 \leq j \leq 5), \lambda_{1}+\lambda_{2}+\lambda_{3} \notin \mathbb{Z}_{>0}$. For such weights, $\beta \mathbf{n b c}(\mathcal{T})=\left\{\eta_{2,4}, \eta_{3,4}\right\}$, and $\beta \mathbf{n b c}(\mathcal{G})=\left\{\eta_{2,3}, \eta_{2,4}, \eta_{3,4}\right\}$. The projection $P(\mathcal{T}): H^{2}(\mathcal{G}) \rightarrow H^{2}(\mathcal{T})$ has matrix

$$
P(\mathcal{T})=\left(\begin{array}{cc}
\frac{-\lambda_{3}}{\lambda_{1}+\lambda_{2}+\lambda_{3}} & \frac{\lambda_{2}}{\lambda_{1}+\lambda_{2}+\lambda_{3}} \\
1 & 0 \\
0 & 1
\end{array}\right)
$$

The arrangements $\mathcal{A}_{j}$ in Figure 11 represent types $\mathcal{T}^{\prime}=\mathcal{T}_{j}$ corresponding to irreducible components of the divisor $\mathrm{D}(\mathcal{T})=\overline{\mathrm{B}}(\mathcal{T}) \backslash \mathrm{B}(\mathcal{T})$. The Gauss-Manin connection matrices $\Omega_{\mathcal{T}}\left(\mathcal{T}_{j}\right)$ are determined by Theorem 7.3 as follows.

$$
\begin{aligned}
& \operatorname{dep}\left(\mathcal{T}_{1}, \mathcal{T}\right)=\{345\}, m_{345}=1, \Omega_{\mathcal{G}}(345)=\left(\begin{array}{ccc}
0 & 0 & -\lambda_{2} \\
0 & 0 & \lambda_{2} \\
0 & 0 & -\lambda_{1}-\lambda_{2}
\end{array}\right), \\
& \Longrightarrow \Omega_{\mathcal{T}}\left(\mathcal{T}_{1}\right)=\left(\begin{array}{cc}
0 & \lambda_{2} \\
0 & -\lambda_{1}-\lambda_{2}
\end{array}\right) .
\end{aligned}
$$

(2) $\operatorname{dep}\left(\mathcal{T}_{2}, \mathcal{T}\right)=\{124,125\}, m_{124}=m_{125}=1, \Omega_{\mathcal{G}}(125)=\left(\begin{array}{ccc}-\lambda_{3} & -\lambda_{3} & 0 \\ -\lambda_{4} & -\lambda_{4} & 0 \\ 0 & 0 & 0\end{array}\right)$,

$$
\Omega_{\mathcal{G}}(124)=\left(\begin{array}{ccc}
0 & 0 & 0 \\
\lambda_{4} & \lambda_{1}+\lambda_{2}+\lambda_{4} & \lambda_{2} \\
0 & 0 & 0
\end{array}\right) \Longrightarrow \Omega_{\mathcal{T}}\left(\mathcal{T}_{2}\right)=\left(\begin{array}{cc}
\lambda_{1}+\lambda_{2} & \lambda_{2} \\
0 & 0
\end{array}\right) .
$$

$$
\begin{aligned}
& \operatorname{dep}\left(\mathcal{T}_{3}, \mathcal{T}\right)=\{124,134,234\}, m_{124}=m_{134}=m_{234}=1, \\
& \Omega_{\mathcal{G}}(134)=\left(\begin{array}{ccc}
0 & 0 & 0 \\
0 & 0 & 0 \\
-\lambda_{4} & \lambda_{3} & \lambda_{1}+\lambda_{3}+\lambda_{4}
\end{array}\right), \Omega_{\mathcal{G}}(234)=\left(\begin{array}{rrr}
\lambda_{4} & -\lambda_{3} & \lambda_{2} \\
-\lambda_{4} & \lambda_{3} & -\lambda_{2} \\
\lambda_{4} & -\lambda_{3} & \lambda_{2}
\end{array}\right) \\
& \Longrightarrow \Omega_{\mathcal{T}}\left(\mathcal{T}_{3}\right)=\left(\begin{array}{cc}
\lambda_{1}+\lambda_{2}+\lambda_{3}+\lambda_{4} & 0 \\
0 & \lambda_{1}+\lambda_{2}+\lambda_{3}+\lambda_{4}
\end{array}\right) .
\end{aligned}
$$

8.3. A Selberg arrangement. We conclude with an example of type $\mathcal{S}$ for which $\mathrm{B}(\mathcal{S})$ has higher codimension in $\overline{\mathrm{B}}(\mathcal{G})$. Let $\mathcal{S}$ be the combinatorial type of the Selberg arrangement $\mathcal{A}$ in $\mathbb{C}^{2}$ with defining polynomial

$$
Q(\mathcal{A})=u_{1} u_{2}\left(u_{1}-1\right)\left(u_{2}-1\right)\left(u_{1}-u_{2}\right)
$$

depicted in Figure 2. See [1, 21, 15] for detailed studies of the Gauss-Manin connections arising in the context of Selberg arrangements.

A collection of weights $\lambda$ is $\mathcal{S}$-nonresonant if

$$
\lambda_{j}(1 \leq j \leq 6), \lambda_{1}+\lambda_{2}+\lambda_{6}, \lambda_{1}+\lambda_{3}+\lambda_{5}, \lambda_{2}+\lambda_{4}+\lambda_{5}, \lambda_{3}+\lambda_{4}+\lambda_{6} \notin \mathbb{Z}_{\geq 0} .
$$




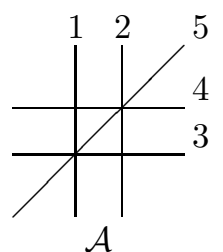

$\mathcal{A}$

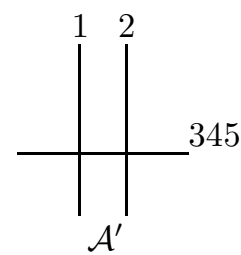

345

Figure 2. A Selberg Arrangement and One Degeneration

For such weights, $\beta \mathbf{n b c}(\mathcal{S})=\left\{\eta_{2,4}, \eta_{2,5}\right\}, \beta \mathbf{n b c}(\mathcal{G})=\left\{\eta_{i, j} \mid 2 \leq i<j \leq 5\right\}$, and the projection $P(\mathcal{S}): H^{2}(\mathcal{G}) \rightarrow H^{2}(\mathcal{S})$ is given by

$$
P(\mathcal{S})\left(\eta_{i, j}\right)= \begin{cases}-\eta_{2,4}-\eta_{2,5} & \text { if }\{i, j\}=\{2,3\}, \\ \eta_{i, j} & \text { if }\{i, j\}=\{2,4\} \text { or }\{2,5\}, \\ 0 & \text { if }\{i, j\}=\{3,4\}, \\ \frac{\left(\lambda_{3} \lambda_{5}-\lambda_{2} \lambda_{5}\right) \eta_{2,4}-\left(\lambda_{2} \lambda_{3}+\lambda_{3} \lambda_{4}+\lambda_{2} \lambda_{5}\right) \eta_{2,5}}{\lambda_{2}\left(\lambda_{1}+\lambda_{3}+\lambda_{5}\right)} & \text { if }\{i, j\}=\{3,5\}, \\ \frac{-\lambda_{5} \eta_{2,4}+\lambda_{4} \eta_{2,5}}{\lambda_{2}} & \text { if }\{i, j\}=\{4,5\} .\end{cases}
$$

The arrangement $\mathcal{A}^{\prime}$ in Figure 2 represents one type $\mathcal{S}^{\prime}$ covered by $\mathcal{S}$. Here $\operatorname{dep}\left(\mathcal{S}^{\prime}, \mathcal{S}\right)=\{134,145,234,235,345,356,456\}$. Since lines 3,4 , and 5 coincide in type $\mathcal{S}^{\prime}$, the order of vanishing of the restriction of $\Delta_{345}$ to $\overline{\mathrm{B}}(\mathcal{S})$ along $\mathrm{B}\left(\mathcal{S}^{\prime}\right)$ is $m_{345}=2$. For all other $J \in \operatorname{dep}\left(\mathcal{S}^{\prime}, \mathcal{S}\right)$, we have $m_{J}=1$. Theorem 7.3 yields

$$
\Omega_{\mathcal{S}}\left(\mathcal{S}^{\prime}\right)=\left(\begin{array}{cc}
\lambda_{3}+\lambda_{4}+\lambda_{5} & 0 \\
0 & \lambda_{3}+\lambda_{4}+\lambda_{5}
\end{array}\right) .
$$

\section{REFERENCES}

[1] K. Aomoto, Gauss-Manin connection of integral of difference products, J. Math. Soc. Japan 39 (1987), 191-208. MR 88f:32031

[2] K. Aomoto, M. Kita, Hypergeometric Functions (in Japanese), Springer-Verlag, Tokyo, 1994.

[3] D. Cohen, Cohomology and intersection cohomology of complex hyperplane arrangements, Adv. Math. 97 (1993), 231-266. MR 94a:32055

[4] D. Cohen, P. Orlik, Arrangements and local systems, Math. Res. Lett. 7 (2000), 299-316. MR 2001i:57040

[5] Some cyclic covers of complements of arrangements, Topology Appl. 118 (2002), 3-15. CMP 2002:08

[6] Gauss-Manin connections for arrangements, Compositio Math., to appear. math.AG/0105063

[7] D. Cohen. A. Suciu. On Milnor fibrations of arrangements, J. London Math. Soc. 51 (1995), 105-119. MR 96e:32034

[8] P. Deligne, Equations Différentielles à Points Sinquliers Réquliers, Lect. Notes in Math., vol. 163, Springer-Verlag, Berlin-New York, 1970. MR 54 \#5232

[9] A. Dimca, Sinqularities and Topology of Hypersurfaces, Universitext, Springer-Verlag, New York, 1992. MR 94b:32058

[10] H. Esnault, V. Schechtman, V. Viehweg, Cohomology of local systems on the complement of hyperplanes, Invent. Math. 109 (1992), 557-561. Erratum, ibid. 112 (1993), 447. MR 93g:32051

[11] M. Falk, H. Terao, $\beta$ nbc-bases for cohomology of local systems on hyperplane complements, Trans. Amer. Math. Soc. 349 (1997), 189-202. MR 97g:52029

[12] I. M. Gelfand, General theory of hypergeometric functions, Soviet Math. Dokl. 33 (1986), 573-577. MR 87h:22012

[13] M. Goresky, R. MacPherson, Stratified Morse Theory, Ergeb. Math. Grenzgeb., vol. 14, Springer-Verlag, Berlin-New York, 1988. MR 90d:57039 
[14] H. Kanarek, Gauss-Manin connection arising from arrangements of hyperplanes, Illinois J. Math. 44 (2000), 741-766. CMP 2000:06

[15] J. Kaneko, The Gauss-Manin connection of the integral of the deformed difference product, Duke Math. J. 92 (1998), 355-379. MR 99h:32024

[16] S. Kobayashi, Differential qeometry of complex vector bundles, Princeton Univ. Press, Princeton, NJ, 1987. MR 89e:53100

[17] P. Orlik, H. Terao, Arrangements of Hyperplanes, Grundlehren Math. Wiss., vol. 300, Springer-Verlag, Berlin, 1992. MR 94e:52014

[18] _ Arrangements and Hypergeometric Integrals, MSJ Mem., vol. 9, Math. Soc. Japan, Tokyo, 2001. CMP 2001:08

[19] R. Randell, Lattice-isotopic arrangements are topologically isomorphic, Proc. Amer. Math. Soc. 107 (1989), 555-559. MR 90a:57032

[20] V. Schechtman, H. Terao, A. Varchenko, Cohomology of local systems and the Kac-Kazhdan condition for singular vectors, J. Pure Appl. Algebra 100 (1995), 93-102. MR 96j:32047

[21] V. Schechtman and A. Varchenko, Arrangements of hyperplanes and Lie algebra homology, Invent. Math. 106 (1991), 139-194. MR 93b:17067

[22] H. Terao, Moduli space of combinatorially equivalent arrangements of hyperplanes and logarithmic Gauss-Manin connections, Topology Appl. 118 (2002), 255-274. CMP 2002:08

[23] A. Varchenko, Multidimensional Hypergeometric Functions and Representation Theory of Lie Algebras and Quantum Groups, Adv. Ser. Math. Phys., vol. 21, World Scientific, River Edge, NJ, 1995. MR 99i:32029

[24] S. Yuzvinsky, Cohomology of the Brieskorn-Orlik-Solomon algebras, Comm. Algebra 23 (1995), 5339-5354. MR 97a:52023

Department of Mathematics, Louisiana State University, Baton Rouge, LA 70803

E-mail address: cohen@math.1su.edu

$U R L:$ http://www.math.1su.edu/ ${ }^{\sim}$ cohen

Department of Mathematics, University of Wisconsin, Madison, Wi 53706

E-mail address: prlik@math.wisc.edu 